\title{
Pediatric Transplantation in the United States, 1996-2005
}

\author{
S. Horslen ${ }^{a}{ }^{*}$, M. L. Barr ${ }^{b}$, L. L. Christensen ${ }^{c}$, \\ R. Ettenger ${ }^{d}$ and J. C. Magee ${ }^{e}$
}

${ }^{a}$ Children's Hospital and Regional Medical Center, Seattle, Washington, USA

b University of Southern California, Los Angeles, California, USA

'Scientific Registry of Transplant Recipients (SRTR), Arbor Research Collaborative for Health, Ann Arbor, Michigan, USA

${ }^{\mathrm{M}}$ Mattel Children's Hospital at the University of California

Los Angeles, Los Angeles, California, USA

e SRTR, University of Michigan, Ann Arbor, Michigan, USA

* Corresponding author: Simon Horslen,

simon.horslen@seattlechildrens.org

Solid organ transplantation is accepted as a standard lifesaving therapy for end-stage organ failure in children. This article reviews trends in pediatric transplantation from 1996 to 2005 using OPTN data analyzed by the Scientific Registry of Transplant Recipients. Over this period, children have contributed significantly to the donor pool, and although the number of pediatric donors has fallen from 1062 to 900 , this still accounts for $12 \%$ of all deceased donors. In 2005, 2\% of 89884 candidates listed for transplantation were less than 18 years old; in 2005, 1955 children, or $7 \%$ of 28105 recipients, received a transplant. Improvement in waiting list mortality is documented for most organs, but pretransplant mortality, especially among the youngest children, remains a concern. Posttransplant survival for both patients and allografts similarly has shown improvement throughout the period; in most cases, survival is as good as or better than that seen in adults. Examination of immunosuppressive practices shows an increasing tendency across organs toward tacrolimus-based regimens. In addition, use of induction immunotherapy in the form of anti-lymphocyte antibody preparations, especially the interleukin-2 receptor antagonists, has increased steadily. Despite documented advances in care and outcomes for children undergoing transplantation, several considerations remain that require attention as we attempt to optimize transplant management.

Key words: Allocation, immunosuppression, living donors, OPTN, pediatric transplantation, SRTR, survival, waiting list

\section{Introduction}

This article is the fifth in a series of annual reviews presenting data and analysis from the Scientific Registry of Transplant Recipients (SRTR) regarding pediatric solid organ transplantation in the Unites States (1-4). It will present updated trends, discussion of analyses presented during the year by the SRTR to the committees of the Organ Procurement and Transplantation Network (OPTN) and discussion of important issues regarding pediatric organ transplantation raised throughout the past year. Unless otherwise stated, the statistics in this article are drawn from the reference tables of the 2006 OPTN/SRTR Annual Report. In this article, pediatric patients are defined as candidates, recipients or donors aged 17 years or less. Throughout this article, data for both graft and patient survival are reported as unadjusted survival unless otherwise stated (adjusted patient and graft survival are available in the reference tables). Short-term survival (3-month and 1-year) reflects outcomes for transplants performed in 2003 and 2004; 3year survival reflects transplants done from 2001 to 2004; 5-year survival reports on transplants performed from 1999 to 2004. Details on the methods of analysis employed may be found in the reference tables themselves or in the technical notes of the 2006 OTPN/SRTR Annual Report, both available online at http://www.ustransplant.org.

This article, unlike the organ-specific articles in this report, discusses data pertaining to all types of solid organ transplantation in a specific subgroup of candidates, namely children. The considerations particular to pediatric populations have been discussed in the previous SRTR annual reports (1-4), and, in recognition of these issues, the transplant community has generally been willing to provide special consideration for children in terms of allocation policy.

The progress made in pediatric organ transplantation over the last 20 or more years has been well documented in the literature (5-9). However, because of the small size of most pediatric programs, cumulative multicenter data covering all recipients and donors in the United States are of special importance. These data, collected by the OPTN and used for complex analysis and modeling by the SRTR, provide important information for use by the OPTN Pediatric and organ-specific committees, guiding both discussion and modification of organ allocation and outcomes assessment. In addition, the pediatric community 


\section{Horslen et al.}

has developed several other national and international multi-center collaborations, including the North American Pediatric Renal Transplant Cooperative Study (NAPRTCS) and the Studies of Pediatric Liver Transplant (SPLIT), as sources for further in-depth analyses.

\section{Pediatric organ donors}

Pediatric donation has declined somewhat in the past 10 years, both in number (from 1062 donors in 1996 to 900 in 2005) and in the percentage of total donors (from $20 \%$ in 1996 to $12 \%$ in 2005). The distribution of pediatric donor ages has remained consistent, with close to $60 \%$ of pediatric donors aged between 11 and 17 years. Among deceased kidney donors, the number and percentage of pediatric donors declined between 1996 and 2005, from 978 (19\%) to 799 (12\%). The age distribution of pediatric kidney donors has remained consistent. The number of pediatric pancreas donors has increased more slowly than the number of adults donating pancreata; the number of pediatric donors grew from 295 to 391, while the percentage of pediatric donors declined from $23 \%$ to $19 \%$. The age distribution has fluctuated, but the largest group of pediatric pancreas donors has been among ages $11-17$ years (80-91\% each year). While the total number of liver donors has increased over the past 10 years, the number of pediatric liver donors has decreased, from $946(21 \%)$ to $815(12 \%)$. As with other organs, donors aged 11-17 years make up the largest fraction of pediatric liver donors in 2005, at 58\%. The number of pediatric intestine donors has increased from 34 to 133 , but no consistent trend in percentage has been observed. The number of pediatric heart donors has declined faster than the number of adult heart donors, from $620(25 \%)$ to 455 (20\%). The absolute number of pediatric lung donors has not changed substantially, but the percentage of pediatric donors has declined from $24 \%$ to $16 \%$.

The number of organs that become available from pediatric donation after cardiac death (DCD) has been increasing. Beginning next year, all hospitals will need to demonstrate that they are establishing policies and procedures for handling DCD.

\section{Waiting list}

The total number of pediatric candidates on the waiting list increased steadily, from 1621 in 1996 to a peak of 2344 in 2001. The total has since leveled, with 2181 candidates (both active and inactive) in 2005 (Figure 1). Distribution by age of pediatric candidates on the waiting list has remained consistent, with $6 \%$ aged less than 1 year, 27\% 1-5 years, 20\% 6-10 years and 47\% 11-17 years at the end of 2005 . The overall percentage of the waiting list made up of pediatric candidates has declined somewhat from 3\% in 1996 to $2 \%$ in 2005 . Changes in the pediatric waiting list by organ type are illustrated in Figure 2.

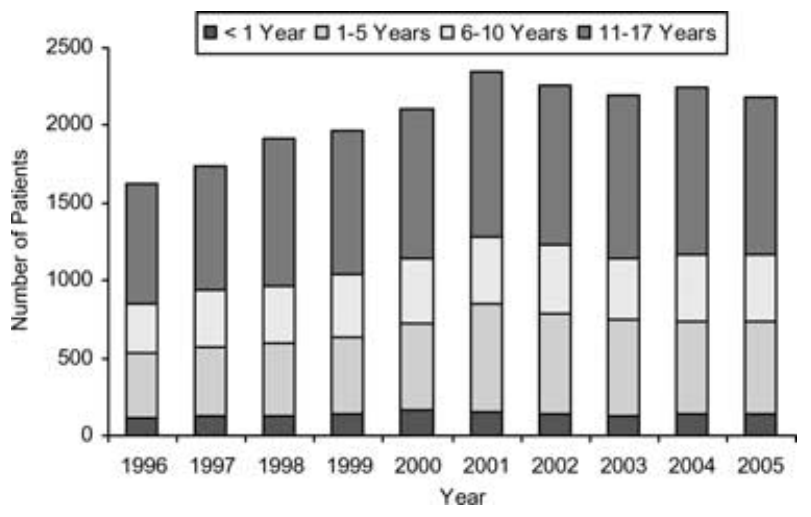

Source: 2006 OPTN/SRTR Annual Report, Table 1.4.

Figure 1: Pediatric transplant waiting list (active and inactive) at year-end for all organs, by age, 1996-2005.

\section{Transplantation and survival}

Over the 10-year period, the number of pediatric recipients of any organ grew 23\% (from 1594 to 1955), in contrast to the $44 \%$ increase in the number of adult recipients (from 18153 to 26150$)$. The largest increase, 30\%, occurred in the largest group, recipients 11-17 years old (661 in 1996 and 861 in 2005). The remaining pediatric groups experienced increases of smaller proportion: recipients 6-10 years old increased $14 \%$ to 308 , recipients $1-5$ years old $18 \%$ to 494 and recipients less than 1-year-old 19\% to 292. As with the waiting list, the age distribution among transplant recipients has remained stable, with $15 \%$ under 1 year, 25\% 1-5 years, 16\% 6-10 years and 44\% $11-17$ years in 2005 . The proportion of pediatric patients among recipients has declined, from 8\% in 1996 to $7 \%$ in 2005.

\section{Kidney Transplantation}

It has become axiomatic that kidney transplantation is the optimal treatment for children with end-stage renal disease

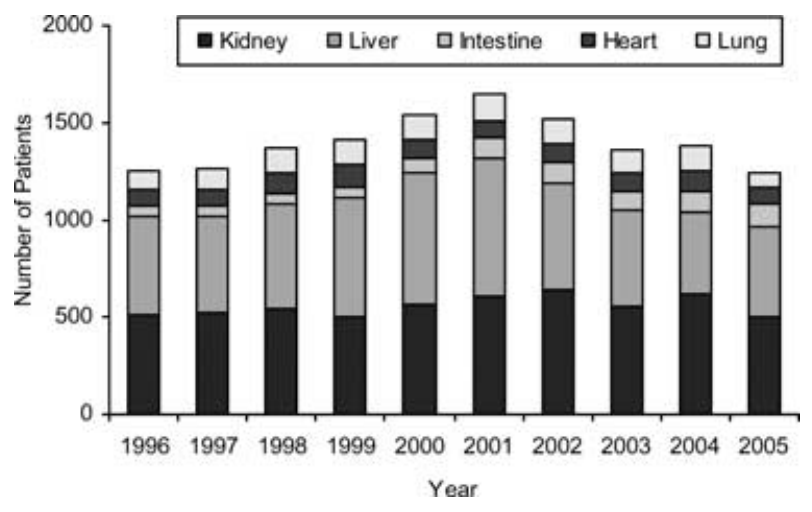

Source: 2006 OPTN/SRTR Annual Report, Tables 5.1a, 9.1a, 10.1a, $11.1 \mathrm{a}$, and $12.1 \mathrm{a}$.

Figure 2: Distribution of active pediatric waiting list candidates at year-end, by organ, 1996-2005. 


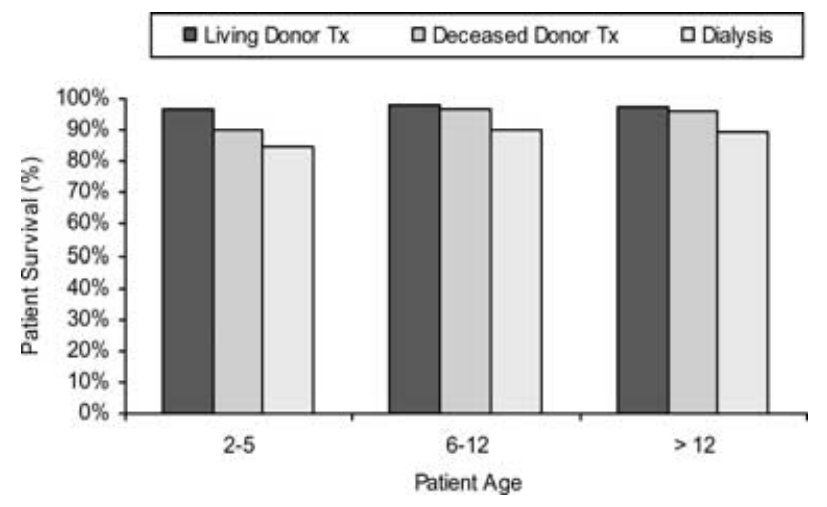

Source: NAPRTCS 2006 Annual Report.

Figure 3: Pediatric patient survival 36 months after kidney transplantation versus dialysis.

(ESRD), and approximately two-thirds of pediatric patients with ESRD will undergo kidney transplantation (10). Numerous studies document the improvements in medical outcomes, cognitive function, social adjustment, sexual maturation and quality of life that accompany kidney transplantation (10).

Perhaps most importantly, survival in pediatric patients with kidney transplants clearly exceeds that seen with dialysis. Data from the 2006 NAPRTCS show that at every age, patient survival at 3 years with either living donor or deceased donor transplantation is markedly superior to that seen in dialysis patients (Figure 3) (11). Even this may understate the value and importance of kidney transplantation in pediatric patients. Recent data from the United States Renal Data System (12) show that at all ages, the years of life expectancy are greater in transplant patients compared with those receiving dialysis, but improvement is clearly greatest in children and adolescents (Figure 4). In children aged 0-14 years, there was an improvement of 30 years; overall, there was an expected calculated remaining lifetime of 50 years for transplant recipients. In patients 15-19

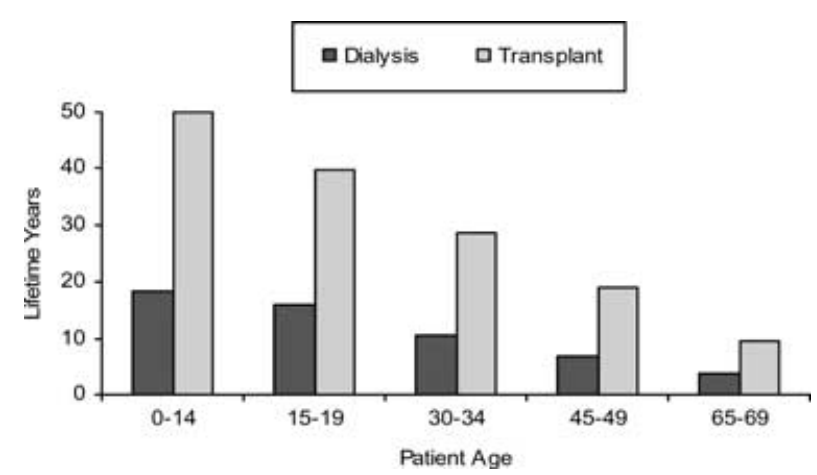

Source: USRDS 2005 Annual Data Report, Table 6b.

Figure 4: Expected remaining lifetimes (years) of dialysis and transplant patients with ESRD, by age.

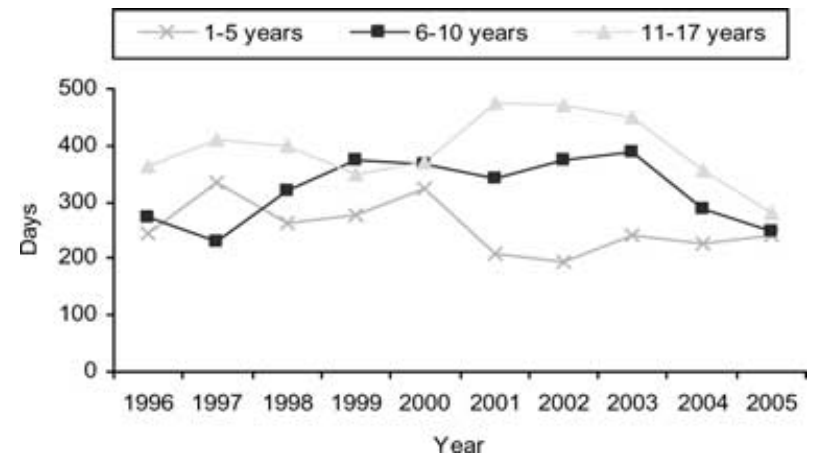

Source: 2006 OPTN/SRTR Annual Report, Table 5.2.

Figure 5: Median time to transplant for new kidney transplant waiting list registrants, by age, 1996-2005.

years old, the improvement in life expectancy for transplant recipients over dialysis patients is approximately 25 years, and the overall calculated remaining lifetime expectancy is 40 years.

\section{Waiting list}

The kidney transplant community and the OPTN have been committed to expediting kidney transplantation for children and adolescents. Within the allocation framework of the OPTN, a number of algorithms have been used in attempts to transplant pediatric patients as promptly as feasible with organs that are optimal, both physiologically and immunologically. Until recently, the generally agreed-upon underlying principle regarding deceased donor allocation has been to try to transplant children from 0 to 6 years old within 6 months, children from 7 to 12 years within 12 months and patients $12-18$ years within 18 months. Over the past 10 years, these efforts have been moderately successful (Figure 5). Since 1998, the youngest children have experienced the shortest median waiting time. Moreover, since 2003, pediatric patients aged $6-10$ years and $11-17$ years have had dramatic decreases in their median waiting time.

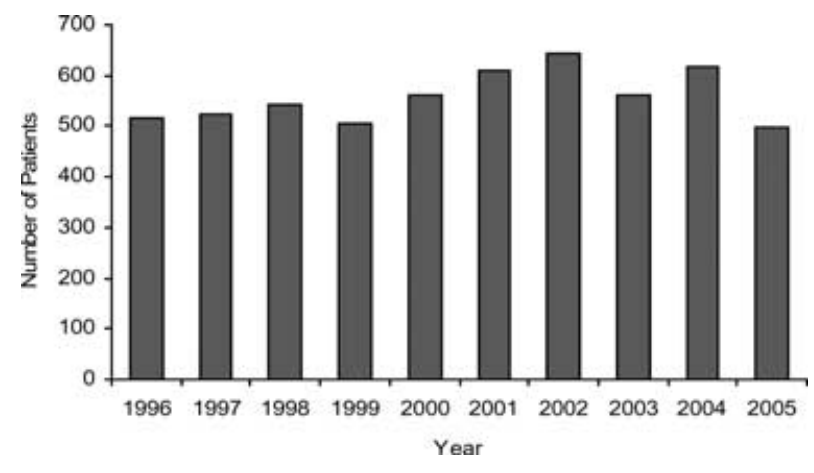

Source: 2006 OPTN/SRTR Annual Report, Table 5.1a.

Figure 6: Active pediatric kidney patients on the waiting list at year-end, 1996-2005. 


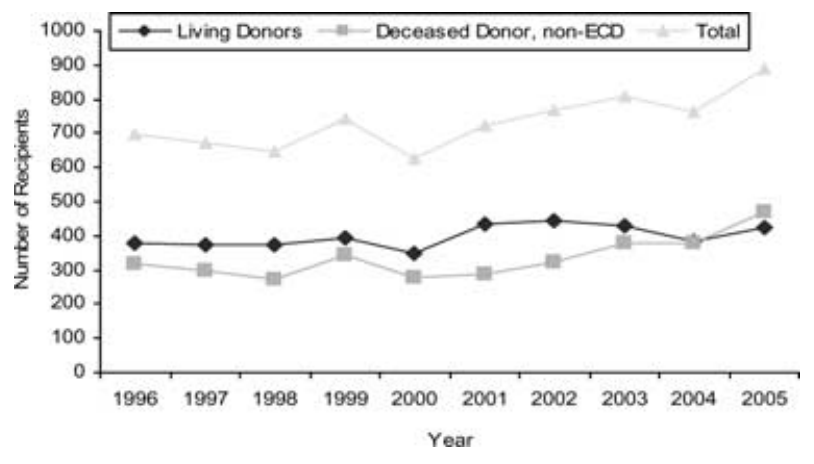

Source: 2006 OPTN/ SRTR Annual Report, Tables 5.4a and 5.4c.

Figure 7: Pediatric kidney transplant recipients, by donor type, 1996-2005.

In 2005, the median waiting time for all pediatric groups was less than 300 days. This compares favorably with the latest data on median waiting time in adults, which over the past 10 years was never less than 920 days in any age group.

Over the past 10 years, the size of the active pediatric waiting list has consistently remained in the range of 500-650 (Figure 6). Parenthetically, the adult waiting list has grown from 28241 in 1996 to 45853 in 2005 . If pediatric patient waiting time is decreasing as the size of the waiting list remains essentially stable, then it seems logical to expect an increase in the number of pediatric kidney transplants performed over this time. There appears to be a trend toward an increasing total number of transplants to pediatric recipients since 2000 (Figure 7), mostly due to an increase in deceased donor transplants. Kidney transplants performed as part of kidney-liver transplantation have consistently remained at approximately $2-5 \%$ of the total number of kidney transplants (Figure 8) (SRTR analysis, May 2006).

\section{Transplantation and survival}

There has been some increase in the number of living donor transplants over this time. However, the number of

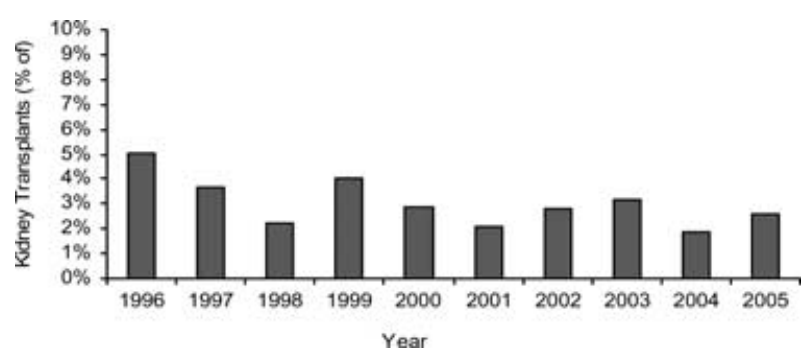

Source: 2006 OPTN/SRTR Annual Report, Table 5.4a and SRTR Analysis, May 2006.

Figure 8: Percentage kidney/liver transplants among deceased donor kidney transplants to pediatric recipients, 19962005.

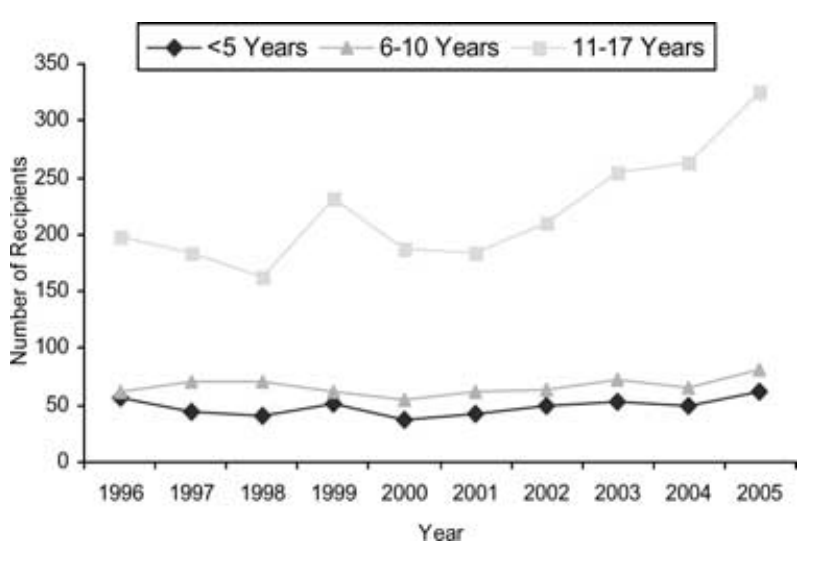

Source: 2006 OPTN/SRTR Annual Report, Table 5.4a.

Figure 9: Pediatric transplant recipients of non-ECD deceased donor kidneys, by age, 1996-2005.

deceased donor transplants has increased markedly, from 278 in 2000 to 468 in 2005 . Historically, in pediatric ESRD patients, the number of living donor transplants has consistently exceeded the number of deceased donor transplants; but 2005 was the first year in a decade that the number of deceased donor transplants exceeded the number of living donor transplants (Figure 7). In contrast, the number of deceased donor transplants to adults has consistently exceeded the number of living donor transplants to adults.

With regard to living donor transplants, the number for each age group has remained essentially the same over the past decade. However, there have been increases in the number of deceased donor transplants in all three pediatric patient age groups (Figure 9). The increases since 2000-2001 in the two younger age groups have been relatively modest, but the increase in the number of patients aged 11-17 years has been quite dramatic. In $2005,70 \%$ of pediatric

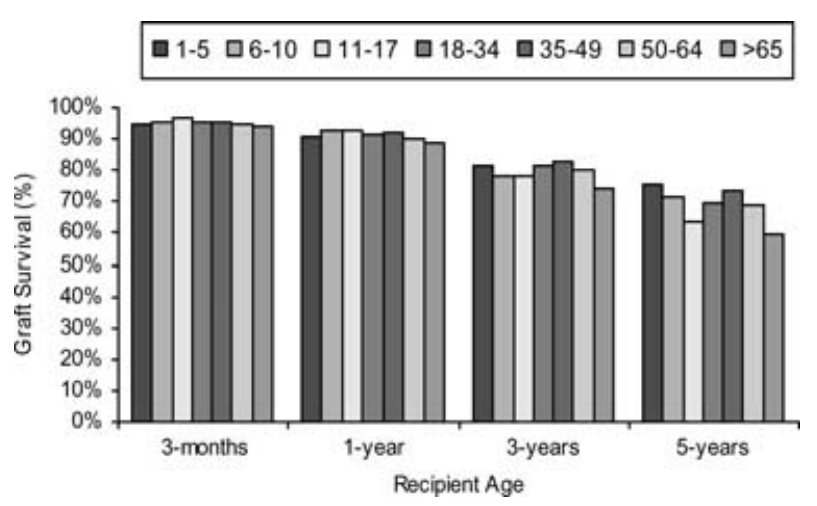

Source: 2006 OPTN/ SRTR Annual Report, Table 5.10a.

Figure 10: Unadjusted 3-month, 1-year, 3-year and 5-year graft survival of deceased donor non-ECD kidney transplants, by recipient age. 


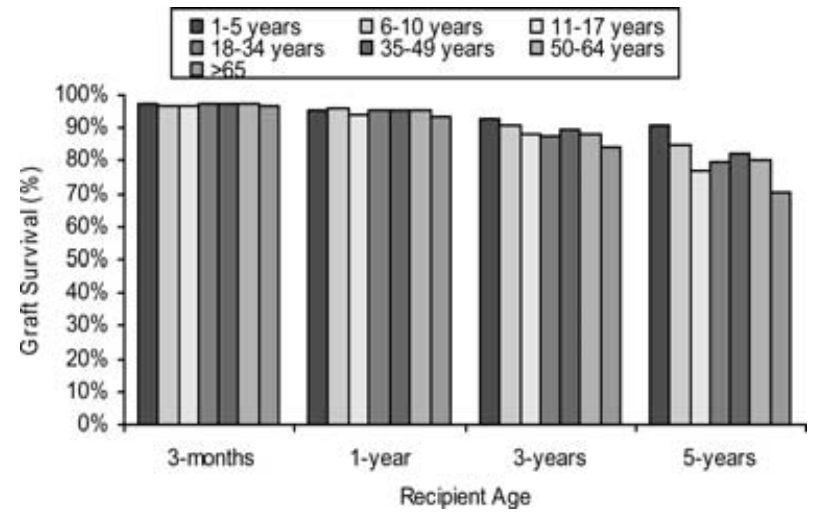

Source: 2006 OPTN/SRTR Annual Report, Table 5.10c.

Figure 11: Unadjusted 3-month, 1-year, 3-year and 5-year graft survival of living donor kidney transplants, by recipient age.

kidney transplants were performed in the 11-17-year-old group.

The traditional OPTN approach for expediting children's access to renal transplantation had been to give extra allocation points to provide them greater priority. It was hoped that these extra points would reflect the desire to offer a reasonable balance between HLA matching and expedited access. Recently, the SRTR, working with the OPTN Kidney-Pancreas Committee, demonstrated that the characteristic most associated with improved outcome in deceased donor pediatric transplantation was donor age between 5 and 35 years (13). Thus in 2005, the OPTN implemented a pediatric kidney allocation policy under which relative priority for kidneys from deceased donors less than 35 years old was assigned to recipients less than 18 years old, after any zero mismatch transplants, recipients with a PRA $>80$, or candidates receiving a kidney with a nonrenal organ (14). While some worry about the lower likelihood of a highly matched kidney under such a policy, Gritsch et al., using OPTN data, independently reported that, except for zero mismatched allografts, the impact of HLA matching in deceased donor pediatric kidney transplantation was minor (15). The increase in pediatric transplant activity in 2005 (Figure 7) suggests that this policy may be having the desired effect, but further analysis will be necessary to confirm this.

Pediatric kidney allograft outcome is excellent, at least in the short term. In all three age groups, results for deceased donor transplants at 3 months and 1 year are as good as those in any other age group (Figure 10). This statement holds true for results at 3 and 5 years for patients aged $1-$ 5 years and $6-10$ years. However, results in patients who were transplanted as adolescents show a greater fall off over 3-5 years. Indeed, at 5 years after transplantation, those patients who were transplanted as adolescents had the poorest allograft outcome of any age group except for recipients aged 65 and older. A similar pattern, although less pronounced, could be observed in recipients of living donor transplants (Figure 11). These are sobering statistics, and, while registry data cannot be used to establish causation, a large body of work suggests that medication nonadherence is likely a major contributor $(16,17)$. While behavioral issues are often offered as the primary factor contributing to nonadherence, another potential variable is the loss of medical insurance that some adolescents may face as they transition to adulthood. The impact of this upon medication nonadherence is unknown; gathering more information related to insurance coverage is important to fully understand this issue.

It has been observed by Watson, among others, that the transition from adolescence to young adulthood is a turbulent and potentially hazardous time for kidney transplant recipients (18). Watson described a small but potentially meaningful experience in England, where it is relatively easy to follow the outcome of pediatric transplant patients into adulthood. He noted that within 15 months of transitioning from pediatric nephrology care to internal medicine, a shocking $30 \%$ of patients had lost their allografts. This phenomenon has become a growing concern for many in pediatric transplantation. However, it is difficult to capture large-scale data on this issue, since pediatric registries such as NAPRTCS cease collecting data when pediatric patients reach adulthood. Because the SRTR can follow outcomes after patients reach 18 years, this database can be a valuable tool in the evaluation of this issue. Attempting to define the problem, Magee et al. have suggested that much of the risk of graft loss in this population begins early in adolescence rather than being solely associated with turning 18 years of age (19).

\section{Immunosuppression}

Multiple reports have described a year-by-year improvement in pediatric renal graft outcome (10). Graft survival has consistently improved, and the number of acute rejection episodes has progressively decreased. There are multiple reasons for these improvements, but changes and improvements in immunosuppression have certainly played an important part. Over the past 10 years, there have been marked changes in the use of immunosuppressive agents in pediatric patients (SRTR analysis, May 2006) (Figure 12). The use of cyclosporine has fallen from almost $80 \%$ in 1996 to less than $15 \%$ in 2005 . In parallel with this, the use of tacrolimus (Prograf ${ }^{\circledR}$, Astellas Pharma U.S., Deerfield, IL) rose from less than $15 \%$ in 1996 to approximately $80 \%$ in 2005. In large part, this switch was engendered by the adverse cosmetic effects of cyclosporine in pediatric patients. Anti-metabolites, increasingly in the form of mycophenolate $\left(\right.$ Cellcept $^{\circledR}$, Roche, Nutley, NJ; Myfortic $^{\circledR}$, Novartis, East Hanover, NJ), were used consistently over the past decade in approximately $80 \%$ of pediatric patients. Over the past 2 years, there has been a decrease in the use of corticosteroids beginning at discharge from 


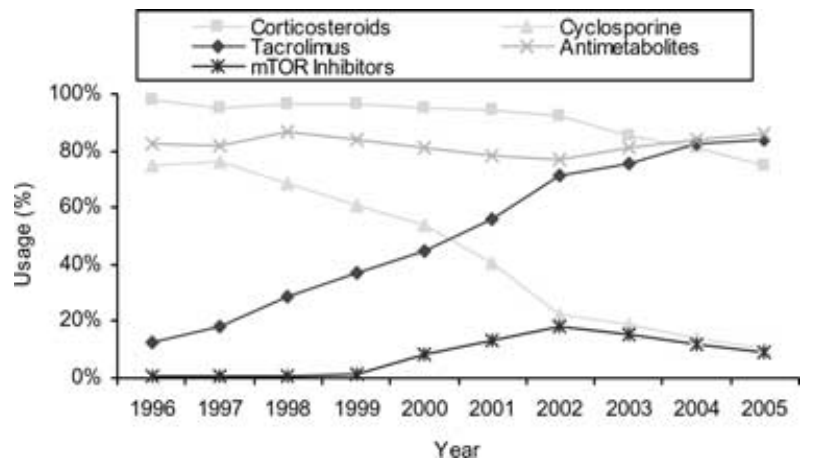

Source: SRTR Analysis, May 2006.

Figure 12: Immunosuppression use for maintenance of pediatric recipients with kidney transplants prior to discharge, 1996-2005.

transplant surgery hospitalization. The side effects of steroids weigh heavily upon pediatric ESRD patients, and so the desire to avoid steroids is understandable. A multicenter, randomized controlled trial of steroid-free immunosuppression has reached full enrollment, and the results are eagerly anticipated.

Figure 13 shows the immunosuppressive combinations that have been initiated in pediatric renal transplant recipients over the past 10 years (SRTR analysis, May 2006). In 2005 , the most popular combination, used in almost $60 \%$ of patients, was tacrolimus, corticosteroids and mycophenolate mofetil (MMF) or mycophenolate sodium. The second most popular regimen in 2005 was the steroid-free combination of tacrolimus and mycophenolate.

The role of biologic agents for induction has been controversial in pediatric kidney transplant recipients. However, use of at least one biologic agent is increasing year by year; Figure 14 shows a constant decrement in pediatric patients with no induction drugs recorded. As a class, the humanized/chimeric anti-CD 25 monoclonal antibod-

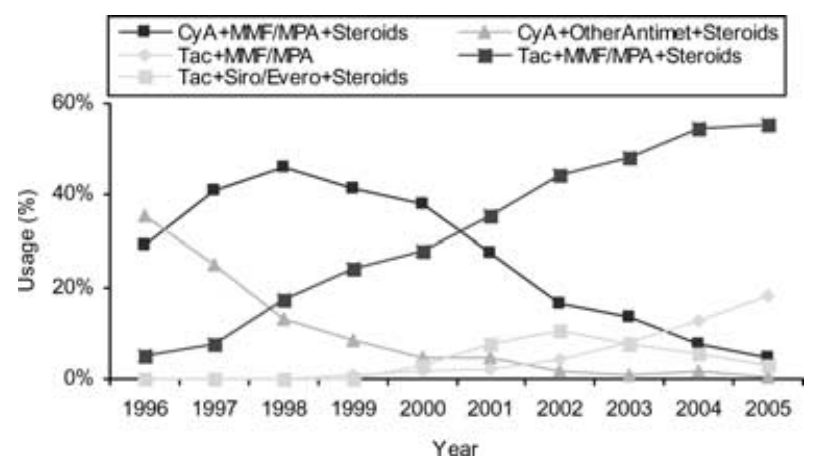

Source: SRTR Analysis, May 2006.

Figure 13: Immunosuppression combinations for maintenance for pediatric recipients with kidney transplants prior to discharge, 1996-2005.

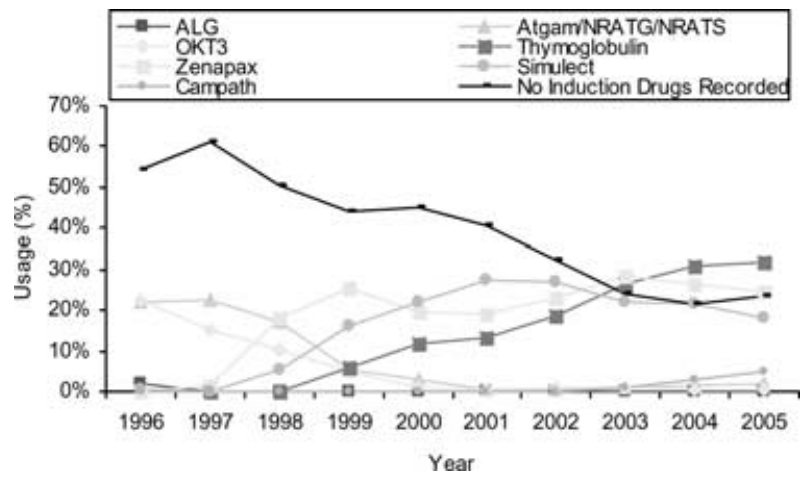

Source: SRTR Analysis, May 2006.

Figure 14: Immunosuppression use for induction for pediatric recipients with kidney transplants, 1996-2005.

ies are most used. However, in the last 2 years, rabbit anti-thymocyte globulin (Thymoglobulin ${ }^{\circledR}$, SangStat Medical Corp., Fremont, CA, 1999) was the induction agent of choice (SRTR analysis, May 2006).

\section{Liver Transplantation}

In the United States, between 1996 and 2005, 5675 children and adolescents received liver transplants. No major technical innovations in liver transplantation occurred during this period, but undoubtedly refinements in surgical technique and improvements in pre- and post-operative care have contributed to continually improving outcomes in terms of survival for both patients and allografts. We are constantly reminded that children differ from adult transplant recipients in terms of size, etiology of organ failure and pharmacokinetics; but in fact these differences also exist within the pediatric population. Congenital causes of liver disease predominate in infants and young children, and an increasing incidence of acquired cholestatic disease appears in older children and adolescents. The peak incidence of liver transplantation in childhood occurs during the first year, dropping sharply in subsequent age groups. Developmental outcomes are of greatest concern in the youngest candidates, but growth and pubertal development remain important considerations until final adult height is achieved. Currently, allocation policy for livers is based on 90-day mortality risk, but there is increasing concern that this may not address the potential for timely liver transplants to avoid irreversible morbidity such as short stature, impaired puberty and cognitive and emotional development issues. These questions of long-term quality outcomes cannot be answered by OPTN data as currently collected, but such data are nonetheless of crucial importance when determining optimal application of liver transplantation for pediatric populations. Welldesigned, prospective, multicenter collaborative data sets, such as those of the SPLIT registry, will be required to guide understanding on these issues. 


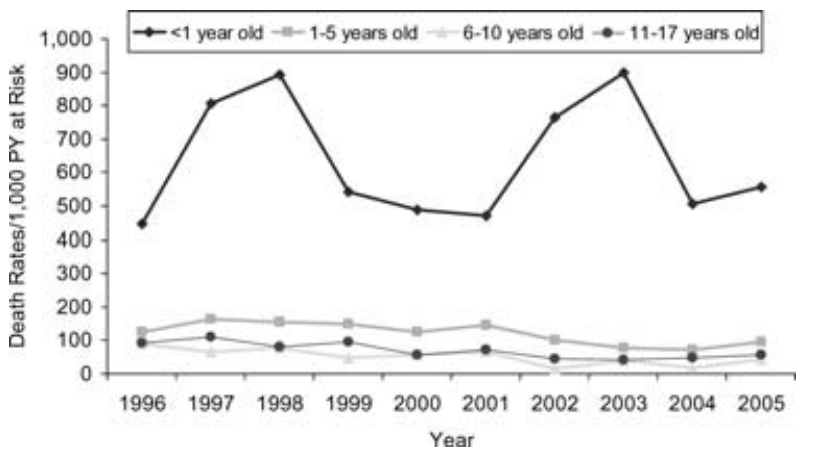

Source: SRTR Analysis, May 2006.

Figure 15: Reported pediatric liver only (no intestine) waiting list deaths and annual death rates per $\mathbf{1 0 0 0}$ patient-years at risk, 1996-2005.

\section{Waiting list}

The number of pediatric patients on the liver waiting list grew steadily, from 498 in 1996 to a peak of 703 in 2001; it has since declined to 462 in 2005 . The sudden decline in new listings of pediatric patients after 2001 clearly reflects the introduction of the Pediatric End-stage Liver Disease (PELD) and Model for End-stage Liver Disease (MELD) scoring systems, which removed the need to list a patient early in order to accrue waiting time. In addition, objectifying waiting list mortality may have enabled clinicians to make sounder judgments on when to list a child with chronic liver disease. Of the children active on the waiting list at the end of 2005, 73 were less than 1 year of age, 170 were $1-5$ years, 83 were $6-10$ years and 136 were 11 years or older. Though the number of pediatric patients on the liver waiting list in 2005 is nearly equal to the number in 1996, pediatric candidates now make up a much smaller proportion of the liver waiting list (4\% compared with $8 \%$ in 1996) because of the large growth in the number of adults active on the liver waiting list, which more than doubled from 5782 in 1996 to 12360 in 2005.

Annual death rates per 1000 patient-years at risk for pediatric candidates on the waiting list for liver only (no intestine) have been variable but displayed a downward trend between 1996 and 2004. However, death rates were higher in 2005 than 2004, at 95 for 1-5-year-olds, 41 for 6-10-year-olds and 58 for 11-17-year-olds (SRTR analysis, May 2006) (Figure 15). Death rates for children less than 1 year old were too variable to distinguish any trend; but, as previously described, this group has the highest death rate while awaiting liver transplantation. Figure 15 demonstrates that this high death rate is not entirely accounted for by infants awaiting combined liver and intestine transplantation. As can be seen, the waiting list mortality rate for infants less than 1 year is between four and eight times that of older children. If we wish to change this high rate, several potential contributing factors will need to be examined to determine causes and possible solutions: (1) etiology of liver failure; (2) timing of referral for transplantation;

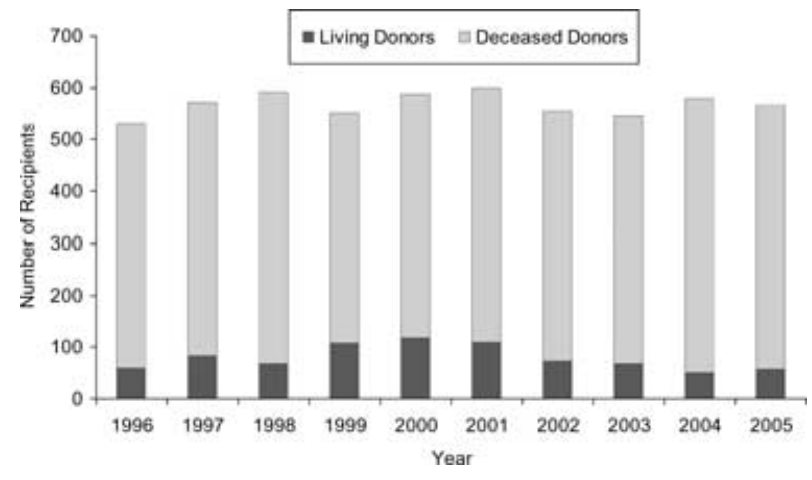

Source: 2006 OPTN/SRTR Annual Report, Tables 9.4a and 9.4b.

Figure 16: Pediatric recipients of deceased and living donor liver transplants, 1996-2005.

(3) appropriate hepatological and intensive care; (4) organ availability; (5) center size and volume and (6) availability of the full range of technical innovations at the listing center, including segmental and living-related liver transplantation. Overall, 101 children died waiting for liver transplantation in 2005, of which 45 were also listed for an intestinal allograft (SRTR analysis, May 2006).

Concerns have been raised about the ability of the PELD score to reliably prioritize children for liver transplantation $(20,21)$. Also, the correlation with MELD scores may be imprecise, making it difficult for children to compete with adult candidates for organs $(20,21)$. Both of these factors have been felt to disadvantage certain candidates in terms of timely access to a liver allograft. The perceived lack of a competitive score has resulted in an excessive number of exception scores being requested from and granted by regional review boards. The use of exception scores, and particularly Status 1 by exception, varied from region to region, but it is probable that this practice undermined the severity scoring systems and further limited their usefulness (22-24). Recent changes in liver allocation policy have attempted to address these issues. The changes to Status 1 limit listing at this most acute status to those who fulfill strict criteria. For a patient outside criteria there is no longer an option to be listed at Status 1 by exception. To gain additional advantage on the waiting list requires prospective submission to local regional review boards for additional PELD or MELD points. In an attempt to guide the regional review boards in granting exception points consistently across the country, a subcommittee of the Liver and Intestine Committee has produced guidelines for several of the more common conditions for which exception scores are requested (23-25). Attempts to create a national review board to achieve patent consistency from region to region have yet to succeed. Although it is too soon to be certain, preliminary analyses of deaths on the waiting list and pediatric transplants, presented to the OPTN Pediatric Committee, suggest that although the number of patients transplanted at Status 1 has inevitably fallen, there 


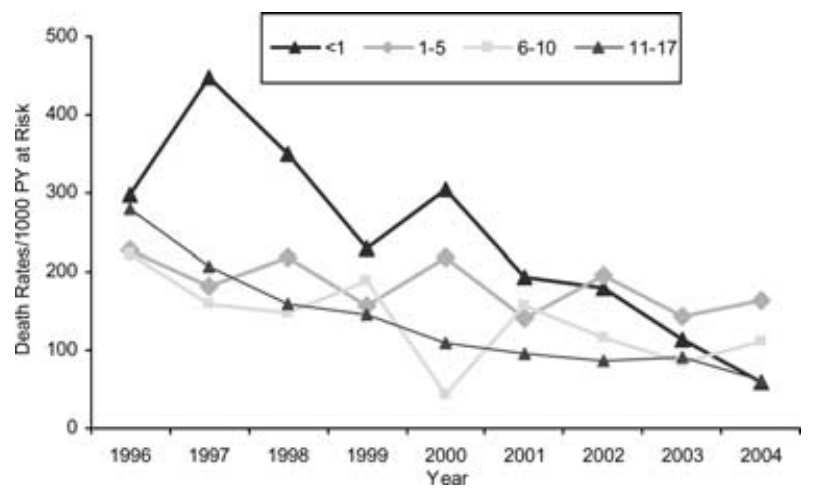

Source: 2006 OPTN/ SRTR Annual Report, Tables 9.7a.

Figure 17: Death rates for pediatric recipients during first year after deceased donor liver transplantation, 1996-2004.

has been no effect on overall pediatric transplant numbers or on pre-transplant mortality rates.

\section{Transplantation and survival}

Figure 16 shows that the total number of pediatric liver transplants per year has been stable in the range of 500 600 procedures annually over the whole period. The number of deceased donor liver transplants to pediatric candidates has ranged from 444 to 529 annually over the 10-year period, ending with 509 recipients in 2005. Living donor recipients peaked in the year 2000 at 118 but diminished to 57 in 2005. The reasons for this decline may be related to the introduction of MELD/PELD, which has enabled sicker patients greater access to deceased donor organs. Did fewer 'not-so-sick' listings, as is suggested by the reduction in the liver waiting list following the introduction of MELD/PELD, allow those in need, but with limited waiting time, access to deceased donor organs? The effect of highly publicized donor deaths in adult-to-adult living donor liver transplantation cannot be discounted, possibly making potential donors or their physicians and surgeons more reluctant to embark on living donor transplantation.

As with death rates on the waiting list, death rates in the first year after deceased donor transplantation have been quite variable over the decade but appear to trend downward, ending at 60 deaths per 1000 patient-years at risk for infants less than 1 year, 164 for 1-5-year-olds, 112 for 6-10-year-olds and 60 for 11-17-year-olds (Figure 17).

Long-term survival is known to be better in children than in adult liver transplant recipients, presumably because of the lack of comorbidities in children. Children are also less likely than adults to have a primary diagnosis associated with significant risk of recurrence leading to graft failure. In 2004, the death rates for every pediatric age group were lower than that for adults aged 18-34 years, even during the first year after deceased donor liver transplantation.

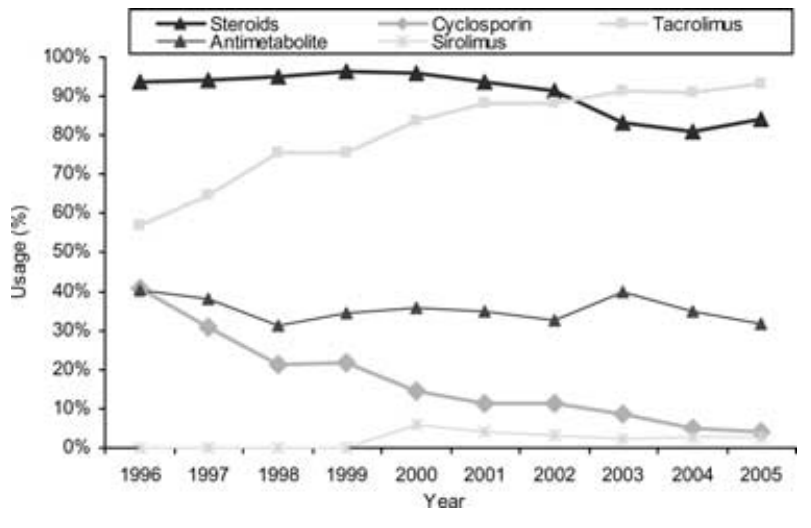

Source: SRTR Analysis, May 2006.

Figure 18: Immunosuppression use for maintenance of pediatric recipients with liver transplants prior to discharge, 19962005.

Among liver recipients under 6 years, graft survival at 1 year was higher for those who received living donor livers than for recipients of deceased donor livers (for less than 1 year: $90 \%$ vs. $84 \%$; for $1-5$ years: $90 \%$ vs. $80 \%)$. The numbers of older children who have received a living donor liver allograft are too small for conclusions to be made about graft and patient survival among the 6-10 and 11-17 year age groups. Unadjusted graft survival among recipients of deceased donor transplants at 1 year was higher for these age groups (86\% for 6-10-yearolds, $88 \%$ for $11-17$-year-olds). Among deceased donor recipients, patient survival at 1 year was best for $11-$ 17 -year-olds at $94 \%$ and worst for $1-5$-year-olds at $90 \%$. Given that the number of liver transplants to adults has increased while the number to pediatric candidates has remained steady, it is not surprising that though the number of pediatric recipients ( $<18$ years at transplant) alive with a functioning liver increased 67\%, from 2554 in 1996 to 4267 in 2004 , their proportion decreased, from $15 \%$ to $13 \%$.

\section{Immunosuppression}

Trends in pediatric maintenance immunosuppression over the last 10 years can be glimpsed from Figure 18 (SRTR analysis, May 2006). Although most pediatric liver transplantation ( $80 \%)$ is done without induction immunosuppression, since 1998 the use of the interleukin-2 receptor (IL-2R) inhibitors basiliximab (Simulect ${ }^{\circledR}$, Novartis, East Hanover, NJ, 2000) and daclizumab (Zenapax ${ }^{\circledR}$, Roche, Nutley, NJ, 1999) in particular has increased (SRTR analysis, May 2006). The use of cytolytic anti-lymphocyte antibody preparations has fluctuated in the $5-10 \%$ range, but the preparation chosen has clearly switched from mostly muromonab-CD3 (OKT3 ${ }^{\circledR}$, Orthobiotech, Bridgewater, NJ) or horse anti-thymocyte globulin $\left(\mathrm{ATGAM}^{\circledR}\right.$, Pharmacia \& Upjohn, Kalamazoo, MI) to predominantly rabbit anti-thymocyte globulin, with a few cases using 
alemtuzumab (Campath-1 $\mathrm{H}^{\circledR}$, ILEX Pharmaceuticals, San Antonio, TX). In the last 5 years, around $90 \%$ of pediatric liver transplant recipients received tacrolimus-based maintenance immunosuppression therapy; cyclosporine use has steadily decreased, from $22 \%$ of patients in 1999 to only $4 \%$ in 2005 . Although steroid-free protocols are frequently discussed, $84 \%$ of pediatric liver transplant recipients were discharged on maintenance corticosteroids in 2005.

\section{Recent changes in liver allocation policy}

Last year saw the introduction of three new allocation policy (14) changes: Share 15 (OPTN Policy 3.6), regional sharing of pediatric donors (OPTN Policy 3.6) and the new Status 1 criteria (OPTN Policy 3.6.4.2). In addition, changes in allocation policy regarding multi-organ transplants that include liver were also adopted.

Share 15 (OPTN Policy 3.6): It was noted that adults on the liver waiting list have a clear survival advantage at 1 year with transplantation if their MELD score was greater than or equal to 18 and a similar risk of death in the first year with or without transplantation between MELD scores of 15 and 18 (26). It was therefore decided to offer livers for transplantation in adults preferentially to recipients locally, then regionally to candidates with MELD scores greater than 15 before offering livers to candidates with scores less than 15. Because there are usually a considerably larger number of adults with high MELD scores awaiting transplantation than pediatric candidates in all regions at any given time, it was feared that such a policy change would lead to a reduction in the number of children transplanted. To attempt to compensate for this policy change, regional sharing of pediatric donors was introduced contemporaneously with Share 15.

Regional sharing of pediatric donor organs (OPTN Policy 3.6): To protect pediatric recipients from effects of Share 15 and in an attempt to increase the use of pediatric organs in pediatric recipients, allocation of livers from deceased pediatric donors was adjusted. The aim was to direct such organs to children awaiting liver transplantation. An early analysis presented recently to the OPTN Pediatric Committee (using only 4 months of data) suggests that following policy implementation there were more transplants performed in recipients aged $0-11$ years from donors aged $0-17$ years but fewer in the 12-17-year age range. A slightly smaller proportion of pediatric donor livers went to adult recipients, but the majority (54\%) of pediatric organs still went to adult recipients. Also of note, more split liver transplants were performed on younger recipients from donors aged less than 18 years, thus making the right side of the liver available for an adult candidate. Caution needs to be applied to the interpretation of this analysis, given the small numbers involved.
This does raise the concern that the policy adjustment is possibly shifting organs away from adolescents to younger children, rather than securing pediatric organs for all children listed. The present allocation protocol offers pediatric organs to $0-11$-year-old patients (i.e. those with PELD scores) before offering them to local adolescents. However, prior to extending offers to regional adolescents, the list is run for local adults and, in this sense, cuts short the pediatric preference at a regional level. This may warrant future committee examination.

New Status 1 criteria (OPTN Policy 3.6.4.2): The revised Status 1 criteria were also introduced in 2005 . The criteria divide the most acute status into two subcatagories, $1 \mathrm{~A}$ and $1 \mathrm{~B}$, the latter classification applying only to children with decompensated chronic liver disease. Strict criteria apply to each of the categories, and patients who do not fulfill the required criteria cannot be listed at Status 1 (A or B) by exception.

Have such changes restricted children's access to liver transplantation? Or, have these policy adjustments simply restructured priority for existing organs within the pediatric population? The answer depends on whether Status 1 by exception candidates were getting organs from the adult pool of deceased donors or whether such candidates were drawing from the existing pediatric donor pool. It is too soon to be certain, but the preliminary analysis, presented to the OPTN Pediatric Committee, of the 2 months prior and subsequent to the implementation of this revision and the previously discussed policy changes fails to show any change in waiting list death rates for children aged 0-11 years and 12-17 years or adults between the two periods.

A number of publications have voiced concern that the present allocation system is not serving children well $(20,21,23)$, but pediatric pretransplant death rates have not risen with the introduction of MELD/PELD nor with any of the subsequent policy modifications. It is possible that the candidates who die on the waiting list may have changed in terms of the severity of diagnosis or geographical distribution from those who died waiting during the pre-MELD era. The present system is predicated on the fact that there is a mortality risk associated with each score. By definition, the severity scoring system can only be validated by predicted mortality rates approximating actual death rates; that is, it is assumed that some children will die. This is hard to accept when (1) there are more pediatric donors than the relatively constant number of potential pediatric recipients each year, and (2) the allocation system(s) have evolved to most equitably deal with the tragic shortage of allografts available for the ever growing numbers of adults with endstage liver disease.

New liver/intestine allocation changes: An SRTR analysis of waiting list mortality comparing candidates of all ages 
listed for liver alone with those listed for a combined liver and intestine transplant showed that the latter group had higher mortality risk. There was no interaction between PELD and the liver/intestine wait-listed candidates, indicating a consistent difference in mortality across PELD scores. The mortality risk among pediatric liver/intestine candidates was 5.5 times higher than that among pediatric liver candidates, which equates to 23 PELD points $195 \%$ Cl 18-28). For patients with a MELD score, the mortality rate difference was smaller, and a clear interaction could be seen between MELD and liver/intestine candidates. Thus, as the MELD score increased, the mortality risk between liver/intestine and liver-alone candidates diminished. At a MELD score of 40 , the waiting list mortality risk for the two groups was essentially equal. In this regard, the current provision of an additional 10\% mortality risk for adult patients on both lists simultaneously appears to work well (SRTR analysis, April 2005).

With strict criteria in place for children with chronic liver disease to upgrade to Status $1 \mathrm{~B}$, it has become obvious that this category was essentially being denied to patients with end-stage liver disease secondary to intestinal failure. The need for positive pressure ventilation or a diminished Glasgow Coma Score are contraindications for combined liver and intestine transplantation in most cases-the patient being too sick to tolerate the procedure. However, portal hypertensive bleeding is common in this patient group, but the pattern of bleeding is different from those patients with variceal bleeding and an intact gut. The current criteria require blood replacement of at least $30 \mathrm{~mL} / \mathrm{kg}$ in a 24-h period. In short bowel patients, the bleeding tends to be persistent and resistant to medical and surgical intervention. Although the bleeding is frequently sufficient to require blood and blood product replacement on a daily basis, rarely is it $30 \mathrm{~mL} / \mathrm{kg}$ in a single day.

Current allocation policy allows, but does not direct, organ procurement organizations (OPOs) to offer composite liver and intestine allografts to patients listed for both organs according to their position on the national intestine list, provided there is no Status 1 liver patient in the region. Feeling uncomfortable with optional routes of organ allocation, the OPTN OPO Committee requested more directive policy. The current policy came into being originally because of the massively high death rate of infants and young children on the waiting list with end-stage liver disease and intestinal failure. The high waiting list death rate is due in large part to the limited number of composite grafts small enough for these infants.

Clearly, an additional advantage is required for children awaiting combined liver and intestine transplantation, and unambiguous allocation policy is to be desired. Therefore, the following suggestions have been submitted by the OPTN Liver and Intestine Committee for public comment and to the OPTN Board of Directors: (1) Children listed simultaneously for liver and intestine transplant would receive an additional 23 PELD/MELD points above their calculated score. (2) For candidates on both the liver and intestine lists, gastrointestinal bleeding necessitating transfusion of $10 \mathrm{~mL} / \mathrm{kg}$ or greater in the preceding $24 \mathrm{~h}$ would enable upgrading to Status 1B. (3) Small composite liver and intestine allografts would be preferentially directed to candidates needing both organs. The specific recommendation is that all combined liver and intestine organs from donors less than 11 years would be offered first to regional Status 1 patients, then to regional pediatric liver candidates with a PELD score of greater than 20 , and then to pediatric candidates at the national level listed for combined liver and intestine transplantation and ranked according to their PELD score.

While the preferential allocation of whole liver grafts to these small liver and intestine recipients will likely improve waiting list mortality in this very ill population, it is noteworthy that such a policy also likely will result in more technical variant grafts in the similarly sized liver-alone recipients. Given that such technical variant grafts are associated with a higher rate of graft loss $(27,28)$, it will be vital to examine the net effect of these policies across all children with liver disease.

\section{Intestine Transplantation}

\section{Waiting list}

The number of pediatric patients active on the intestine waiting list more than doubled, from 53 in 1996 to 116 in 2005; however, the proportion of children on the list remains stable, making up $77 \%$ of the total intestine candidates in 2005. The 25th percentile of time-to-transplant has varied, with no consistent trends for pediatric patients on the intestine waiting list, from 115 to 208 days for candidates less than 1 year (with a single outlier of 894 days in 1997), 46 to 119 days for $1-5$-year-olds, 15 to 183 days for 6-10-year-olds and 4 to 226 days for 11-17-year-olds. Median time-to-transplant is often not available for younger children because fewer than $50 \%$ of these children have been transplanted.

Annual death rates on the intestine waiting list have varied extensively with the highest waiting list death rates of any group of solid organ transplant candidates seen among infants ( $<1$ year old), most of whom are awaiting combined liver and intestine transplantation. The more modest death rates seen in the children over 5 years of age reflect that many more of the older children are listed for intestine transplantation alone; that is, they do not have liver failure. As was documented by Sweet et al. in last year's OPTN/SRTR Annual Report (4), 92\% of deaths on the intestine transplant waiting list are in patients with chronic liver failure disease. The number of children dying on the intestine waiting list has shown no consistent trend, but increased overall from 20 in 1996 to 47 in 2005. Death rates for these children are unacceptably high, and 


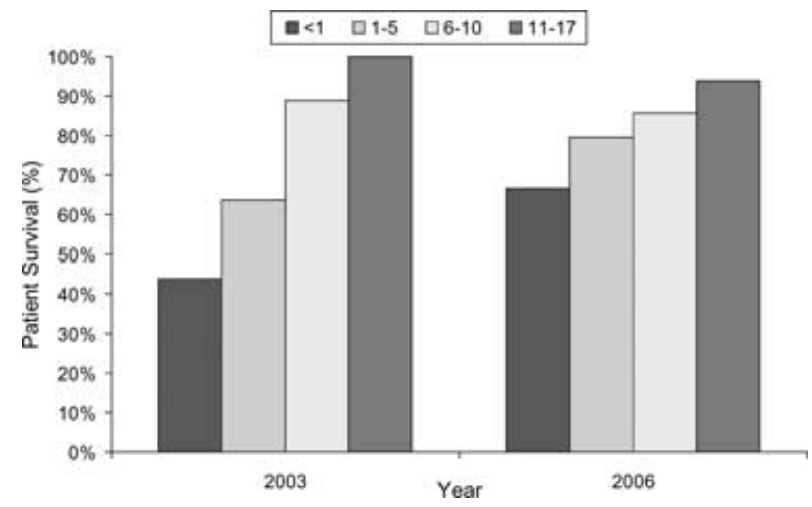

2003 data includes patients transplanted 2000-2001; 2006 data includes patients transplanted 2003-2004.

Source: 2006 OPTN/SRTR Annual Report, Table 10.14 and 2004 OPTN/SRTR Annual Report, Table 10.11.

Figure 19: Unadjusted 1-year patient survival for intestine transplants, by age, for 2003 and 2006.

attempts to address this problem have been discussed in OPTN committees at length (see New Liver/Intestine Allocation Changes section above).

\section{Transplantation and survival}

The number of intestine transplants in pediatric recipients has grown, from 28 in 1996 to 94 in 2005. The incidence of intestine transplants per 1 million population continues to increase, and there is no suggestion that this is likely to plateau in coming years. The increasing numbers of patients considered suitable for intestine transplantation most likely relate to a number of issues. Greater access for patients with irreversible intestinal failure has occurred because of both growth in established intestine transplant programs and the development of new programs. There is an increasing familiarity among referring physicians regarding the possibilities of intestinal transplantation and recognition that quality outcomes are possible. Finally, there also may be a change in approach to abdominal catastrophes, with surgeons, particularly those in neonatal practice, becoming more willing to undertake extensive intestinal resection now that a potential treatment option for the complications of intestinal failure is available. There does not, as yet, appear to be a relaxing of indications for intestine transplantation, but this may, and probably will, occur as the survival figures for this procedure continue to improve.

Improved short-term patient survival following intestine transplantation can be seen by comparing 1-year survival (Figure 19) from the most recent cohort analyzed in the SRTR data set with that of the data presented in the 2003 OPTN/SRTR pediatric report (29).

Graft survival at 5 years for intestine recipients is $42 \%$ for those less than 1 year, $48 \%$ for $1-5$-year-olds, $60 \%$ for 6 10 -year-olds and $40 \%$ for $11-17$-year-olds. Patient survival

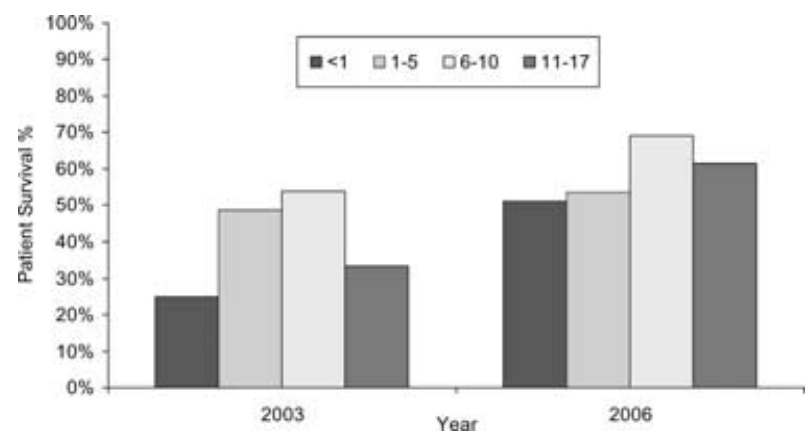

2003 data includes patients transplanted 1996-1997; 2006 data includes patients transplanted 1999-2004.

Source: 2006 OPTN/SRTR Annual Report, Table 10.14 and 2004 OPTN/SRTR Annual Report, Table 10.11.

Figure 20: Unadjusted 5-year patient survival for intestine transplants, by age, for 2003 and 2006.

at 5 years (Figure 20) was $51 \%$ for less than 1 -year-olds, $53 \%$ for $1-5$-year-olds, $69 \%$ for $6-10$-year-olds and $61 \%$ for 11-17-year-olds. These 5-year survival figures represent outcomes for the cohort of patients transplanted several years ago. It is to be hoped that the improvements seen in 1-year survival will in time translate into improved 5year survival for the current cohort of intestine transplant recipients. At the end of 2005, there were 265 pediatric intestine recipients living with functioning transplants.

\section{Immunosuppression}

The data reported on induction drugs given are clearly incomplete, as all the major intestine transplant programs have been using induction regimens over the past few years, employing either anti-lymphocyte preparationspredominantly rabbit anti-thymocyte globulin but also alemtuzumab in older children-or IL-2R antagonists (antiCD25) basiliximab or daclizumab (30). However, the data as available through the OPTN show no induction drugs recorded in over $60 \%$ of pediatric cases in 2005.

Tacrolimus remains the mainstay of maintenance immunosuppression, with between $90 \%$ and $100 \%$ of recipients receiving this drug prior to discharge between 1996 and 2005 (SRTR analysis, May 2006). In fact, 100\% of recipients with reported immunosuppression data during the 10-year period have received tacrolimus at some time between discharge and the end of their first year following transplantation. Cyclosporine has not been used as a primary maintenance immunosuppressant since 2000, but occasional recipients have received the drug later during their first year after transplantation; usage fell from $19 \%$ in 1995 to $2 \%$ in 2004 . The use of adjunctive anti-metabolite medication as part of the primary immunosuppressive regimen has decreased from 67\% (MMF 42\% and azathioprine $25 \%$ ) in 1996 to $14 \%$ in 2005, all of which was MMF. The mTOR inhibitor sirolimus (Rapamune ${ }^{\circledR}$, Wyeth, Philadelphia, PA, 1999) has been introduced during the last 10 years and was first used in intestine recipients in 1999. In 2005, 
sirolimus was given to $11 \%$ of patients prior to discharge and to $23 \%$ at some time up to 1 year following discharge.

In summary, the number of patients referred for and undergoing intestine transplantation continues to increase. Waiting list death rates remain much too high, particularly for the smallest children who are at the greatest risk of rapidly progressive liver disease. The differences in disease etiology, age and waiting list mortality between those candidates requiring an intestine allograft alone or in combination with a liver would suggest that it is time to consider routinely analyzing these patients as two separate groups, akin to the analysis for combined heart and lung transplantation. Attempts are being considered that may direct small donor livers (if suitable for use as a composite allograft with intestine) to the infants listed for both organs rather than to children requiring a liver transplant alone. Short-term survival following intestine transplantation is approaching that of other forms of solid organ transplantation. Improvements in longer term outcomes are to be expected, but assessing outcomes such as growth and development will require data sets other than those collected for SRTR analysis.

\section{Heart Transplantation}

\section{Waiting list}

Although the number of candidates on the heart waiting list has decreased from 2436 in 1996 to 1334 in 2005, the number of pediatric candidates has not changed greatly, with 89 active on the waiting list at the end of 2005 . While the number of new pediatric registrants on the heart waiting list has shown no consistent trend, the number of transplants to pediatric recipients has increased, from 262 in 1996 to 313 in 2005; the number of pediatric deaths on the waiting list has decreased slightly, with 80 deaths in 2005 (Figure 21). Infants $<1$ year and children 11-17 years have accounted for the majority of new registrations in each of the last 10 years (Figure 22). The smallest number of new registrations was in the 6-10-year age group.

\section{Transplantation and survival}

Unadjusted 1-year patient survival for pediatric heart recipients increases with increasing age, from $82 \%$ for children less than 1 year old to $94 \%$ for those 11-17 years. In contrast, unadjusted 5-year patient survival decreased with increasing age, from $75 \%$ among children less than 1 year to $72 \%$ among children 11-17 years (Figure 23). The prevalence of pediatric heart recipients alive with a functioning graft followed the overall trend of all heart recipients, increasing from 1225 at the end of 1996 to 2093 at the end of 2004 (Figure 24).

For survivors of pediatric heart transplantation, the inherent challenges of both short- and long-term care involve prevention of graft vasculopathy (retransplantation may ultimately be required for lifetime care of this population in

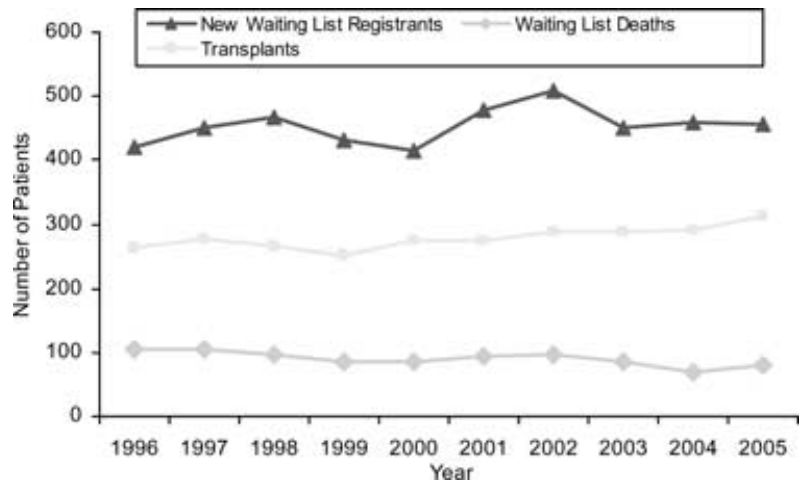

Source: 2006 OPTN/SRTR Annual Report, Tables 11.3-11.4, and 15.3.

Figure 21: New pediatric heart waiting list registrants, deaths on the waiting list and transplants, 1996-2005.

view of their younger age at the time of the primary transplant); prevention and treatment of infection and malignancy; and minimization of end-organ toxicities secondary to chronic long-term complications of chronic immunosuppressive medications.

Renal dysfunction following cardiac transplantation: One of the greatest concerns regarding the development of end-organ toxicity is the decline in renal function seen late after heart transplantation with the current calcineurin inhibitor-based immunosuppressive regimens (31). Increasing numbers of children will require kidney transplantation over the next decade following extra-renal solid organ transplantation in childhood.

A recent study of pediatric heart recipients in the United States from 1990 to 1999 who survived at least 1 year after transplant found a 10 -year actuarial risk of $4 \%$ for

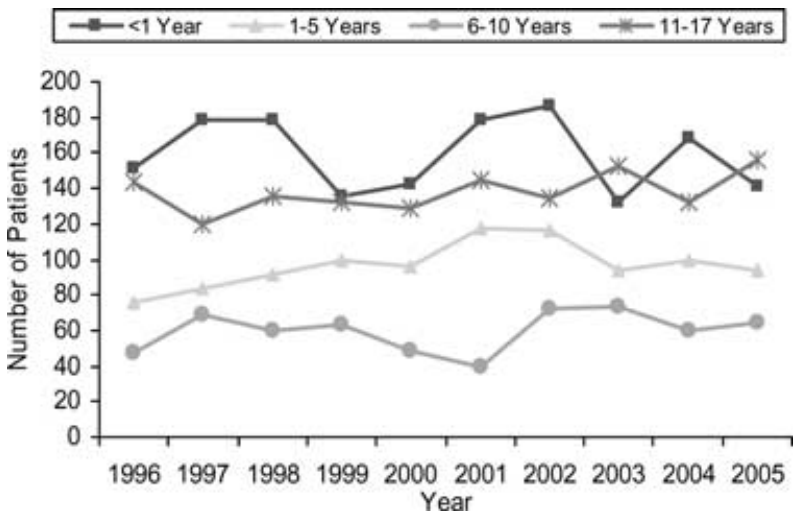

Source: 2006 OPTN/SRTR Annual Report, Table 15.3.

Figure 22: New pediatric registrants on the heart waiting list, by age, 1996-2005. 


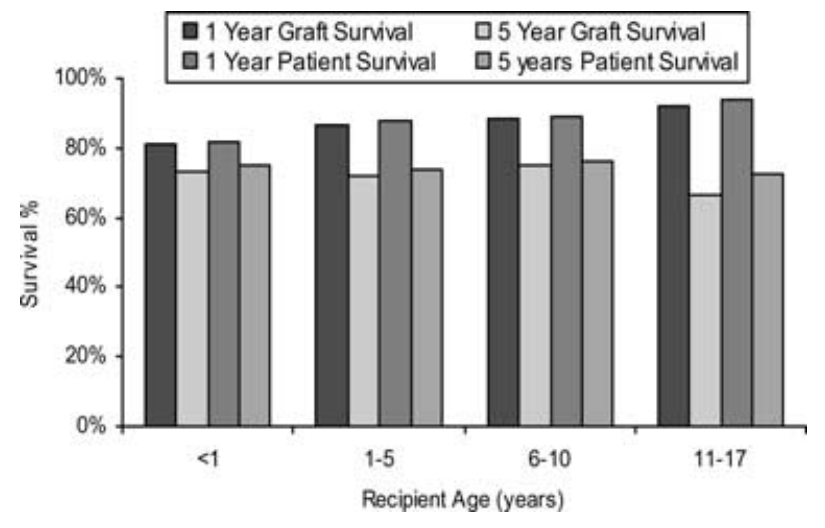

Source: 2006 OPTN/SRTR Annual Report, Tables 11.10 and 11.14 .

Figure 23: Unadjusted 1- and 5-year graft and patient survival of heart transplants, by recipient age.

developing ESRD (defined as chronic dialysis and/or kidney transplant) and a 10 -year actuarial risk of $12 \%$ for developing chronic renal insufficiency $(\mathrm{CRl}$, defined as creatinine $>2.5 \mathrm{mg} / \mathrm{dL}$, including those with ESRD). Those who developed $\mathrm{CRI}$ had nine times the risk of death of pediatric heart recipients without $C R I(p<0.0001)$ (31). An SRTR analysis showed that during the 10 -year period from 1996 to 2005, 55 pediatric heart transplant recipients were placed on the kidney transplant waiting list and 53 pediatric heart recipients underwent a subsequent kidney transplant (SRTR analysis, May 2006).

Incompatible $A B O$ donors for infant recipients: Children in all age groups have a high risk of death while waiting for a heart transplant, with the annual waiting list death rate being higher in all pediatric age groups compared with all adult candidates for most years. The highest death rate is among infants aged less than 1 year, by greater than a log scale difference (with a death rate of 2465 per 1000

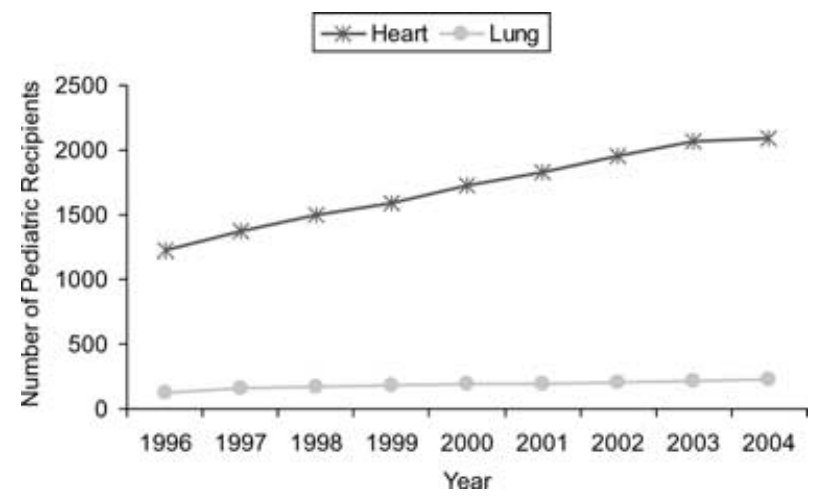

Source: 2006 OPTN/SRTR Annual Report, Tables 11.16 and 12.16.

Figure 24: Prevalence of pediatric recipients living with a functioning transplant at end of year, 1996-2004.

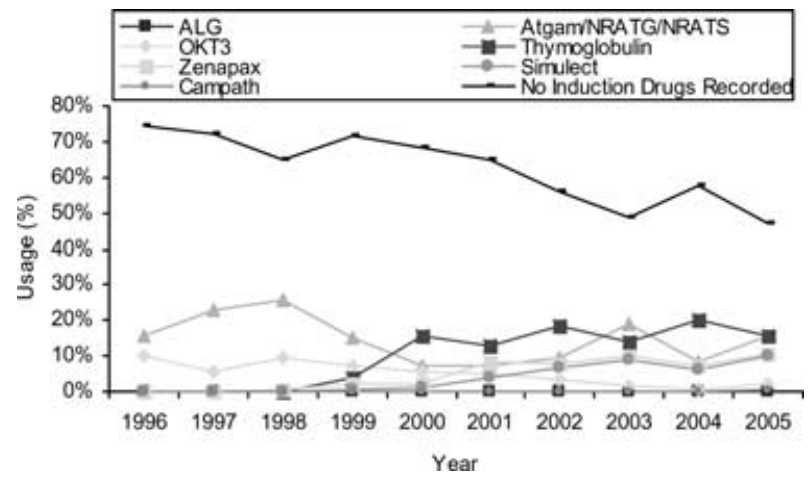

Source: SRTR Analysis, May 2006.

Figure 25: Immunosuppression use for induction for pediatric recipients with heart transplants, 1996-2005.

patient-years at risk vs. 222-229 in children between 1 and 17 years and vs. 128-169 in candidates aged 18 years and greater). A novel approach to dealing with this high death rate was introduced by the team at Sick Children's Hospital in Toronto (32). As a result of that pioneering work, a policy was put in place in the United States to permit pediatric candidates younger than 1 year who did not yet have blood-type-specific antibodies to be listed for incompatible hearts. Between January 1, 1999, and April 19, 2005, 16 pediatric heart recipients under 1 year have been transplanted in the United States with ABO-incompatible donors (SRTR analysis, June 2006). The ages of the recipients ranged from 0 to 7 months. Seven cases involved blood type $A$ donor into type $O$ recipient, three cases were $B$ into $O$, three cases were $B$ into $A$ and three cases were $A B$ into $O$. All of the recipients were Status $1 \mathrm{~A}$ prior to the transplant. Five patients died following transplantation, four of them within the first year, with an unadjusted posttransplant survival of $75 \%$ at 1 year. Of the 16 recipients, only one experienced graft failure in the absence of mortality. There were 342 Status 1A patients less than 1 year of age receiving transplants from blood-type compatible donors during the same time period. Within that cohort, there were 67 deaths during the first year following transplantation, with a resulting survival rate of $82 \%$. Based on these extremely promising initial results, additional pediatric heart transplant programs are developing blood type incompatible programs. On September 29, 2006, the OPTN Board of Directors approved a policy change that permits the upper age range for the recipient of such a transplant to be extended to 2 years, assuming absence of blood type-specific antibodies.

\section{Immunosuppression}

Induction immunosuppression: The use and composition of an induction regimen in pediatric heart transplant recipients has continued to change over the last decade (SRTR analysis, May 2006) (Figure 25). While the frequency of use remains far below that seen currently in kidney 


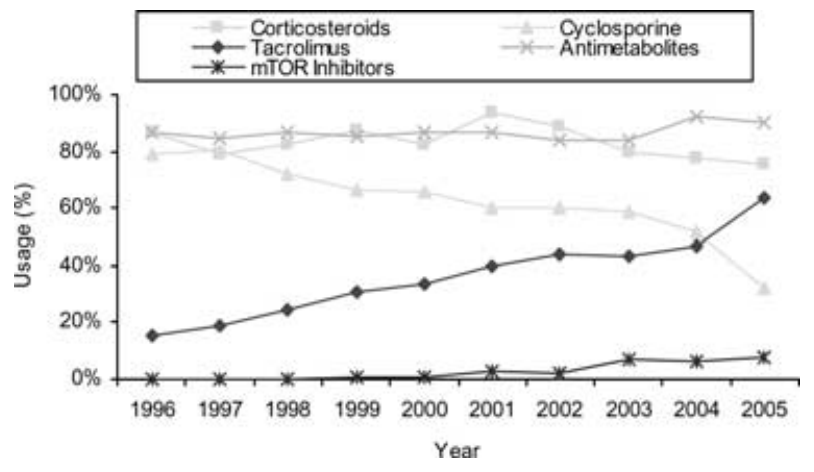

Source: SRTR Analysis, May 2006.

Figure 26: Immunosuppression use for maintenance of pediatric recipients with heart transplants prior to discharge, 1996-2005.

transplantation, there has been a gradual increase, especially in the past 6 years. The rationale for the choice of agent, aside from its use at all, varies by center, and there is a paucity of randomized studies to support these specific choices. During the past decade, the percentage of pediatric heart recipients receiving an induction agent has risen from a low of $25 \%$ in 1996 to a high of $53 \%$ in 2005. In 1998, $73 \%$ of patients who received induction therapy were given anti-thymocyte globulin, compared with only $20 \%$ in 2004. A more dramatic decline has been seen with muromonab-CD3; $40 \%$ of induction patients received it in 1996 versus less than $2 \%$ in 2004 . Practice patterns have changed with the clinical availability of new agents, as concerns have arisen regarding perceived increased risks of vascular rejection (secondary to human-anti-murine antibody development), cytomegalovirus infection (CMV) and posttransplant lymphoproliferative disorders (PTLD). Practice patterns have also shifted regarding use of corticosteroids for maintenance. The use of rabbit anti-thymocyte globulin in pediatric heart transplantation increased from $0 \%$ in 1998 to $30 \%$ of all patients receiving induction in 2005. Similarly, the use of the anti-IL-2R antibodies daclizumab and basiliximab increased from $0 \%$ in 1997 to $39 \%$ in 2005 . The year 2005 marks the first time alemtuzumab has been reported in heart recipients, although usage was negligible.

The use and type of induction therapy also varied depending on whether the recipients were on steroids at the time of discharge from the initial transplant event. Between 2001 and 2005, of the pediatric heart recipients who were steroid-free at discharge, $41 \%$ received induction therapy; rabbit anti-thymocyte globulin represented the single most frequently used induction agent (58\% of all induction use in this group), followed by horse anti-thymocyte globulin $(31 \%)$. For patients who were maintained on steroids (83\% of all pediatric recipients) at discharge, use of induction therapy occurred in less than $40 \%$ of patients; for those in that group who did receive induction, the anti-
IL-2R antibodies were the most commonly used agents (44\% of all induction use in this group) (SRTR analysis, May 2006).

Maintenance immunosuppression: Over the past 10 years, there has been a shift in the agents used for maintenance immunosuppressive therapy prior to discharge (SRTR analysis, May 2006) (Figure 26). Cyclosporine-based regimens have decreased dramatically, from $79 \%$ in 1996 to $32 \%$ in 2005 . Conversely, use of tacrolimus-based regimens has increased, from $16 \%$ in 1996 to $64 \%$ in 2005 , with 2005 being the first year that tacrolimus use exceeded that of cyclosporine. Azathioprine demonstrated an even greater decrease, from $77 \%$ in 1996 to $22 \%$ in 2005, while MMF has become the anti-metabolite of choice, increasing from $8 \%$ in 1996 to $66 \%$ in 2005 . Sirolimus use was first reported in 1999 and reached $8 \%$ in 2005 . While corticosteroids are still employed for the majority of patients, use has declined from a high of $94 \%$ in 2001 to a 10 -year low of $75 \%$ in 2005 .

At the time of discharge in 2005, the single most common regimen was the combination of tacrolimus and MMF in $46 \%$ of pediatric heart transplant recipients, followed by cyclosporine and MMF in $20 \%$ of patients (SRTR analysis, May 2006). The combination of cyclosporine and azathioprine was by far the most common regimen through the 1980s and mid-1990s (63\% in 1996); it is now rarely employed (under $9 \%$ in 2005) (SRTR analysis, May 2006).

In the most recent cohort from 2004, by 1 year after transplantation, use of tacrolimus-based regimens increased to $58 \%$, while use of cyclosporine-based regimens decreased to $38 \%$ (SRTR analysis, May 2006). Of the former, the combination of tacrolimus, MMF and steroids remains the most commonly used. Sirolimus as part of the regimen was used in $6 \%$ of patients at 1 year following transplantation in the 2004 cohort.

The greatest change in maintenance immunosuppression regimens over the first 3 years occurs between discharge and 1 year, presumably in response to rejection, infection and drug side effects (SRTR analysis, May 2006). Ongoing 'regimen attrition' occurs the subsequent years. The highest rate of conservation of the original discharge regimen were seen in the tacrolimus/MMF and cyclosporine/MMF groups, with $56 \%$ and $55 \%$ of patients, respectively, still receiving those combinations 3 years after transplantation. The highest rate of regimen change occurred in the cyclosporine/azathioprine group, of which only $36 \%$ were still receiving it at 3 years.

Steroid withdrawal: In the 2005 cohort, $25 \%$ of patients were corticosteroid-free at discharge from the initial transplant event. This represents a large $(400 \%)$ increase in this practice, compared with only $6 \%$ of patients in 2001 (SRTR analysis, May 2006). In all of the pediatric heart recipients 
in the 2001-2005 cohort, $17 \%$ of recipients were steroidfree at discharge. In that subgroup, $71 \%$ received some form of induction therapy.

Anti-rejection treatment: There has been an overall trend toward reduced use of all types of anti-rejection therapy (SRTR analysis, May 2006). In 2004, 80 patients of a total cohort of 291 received anti-rejection therapy (27\%, down from a 10 -year high of $49 \%$ in 1998) during the first year after transplant. This trend may reflect a true decrease in acute rejection rates associated with the more modern induction and maintenance regimens. Another contributing factor may be that more rejection episodes are being treated with only a change in maintenance regimen agents; decreased rates of rejection may represent an under-reporting of rejection episodes as measured by the use of anti-rejection therapy.

The great majority of patients $(89 \%$ to $98 \%$ over the past 10 years) with reported anti-rejection therapy received corticosteroids as treatment for the episode (SRTR analysis, May 2006). In 2004, 16\% of patients treated for rejection received an anti-lymphocyte antibody preparation; the agent most commonly used was rabbit antithymocyte globulin (61\% of that group), followed by horse anti-thymocyte globulin (23\%). Of interest, 15\% (2 of 13) of the antibody-treated group received an anti-IL-2R antibody, despite little data supporting the use of this class for the treatment of rejection.

\section{Changes in pediatric heart allocation policy}

Until recently, for candidates of all ages, hearts were allocated locally before being offered out to the region. A Status 2 candidate would receive a heart prior to a Status $1 \mathrm{~A}$ or $1 \mathrm{~B}$ candidate waiting outside of the local OPO. However, changes in the heart allocation algorithm have adjusted the role of geography. Once offers to local adult $1 \mathrm{~A}$ and $1 \mathrm{~B}$ candidates are exhausted, the organ is offered to Zone A (centers within 500 miles) Status $1 A$ and $1 B$ candidates before being offered back to local centers for Status 2 candidates.

The pediatric heart transplant community expressed concerns that the new allocation algorithm would disproportionately jeopardize Status 2 pediatric candidates. While adult Status 2 patients can be adequately managed medically and may not derive early benefit from transplantation, similar data are not available for the pediatric population due to the smaller numbers. For this reason, the OPTN Thoracic Committee elected to continue with the local allocation first policy for children.

\section{Heart-Lung Transplantation}

With an average of only seven pediatric heart-lung transplants performed in the United States per year over the past 10 years, it is difficult to make any definitive statements regarding changing patterns. The challenges in pedi-

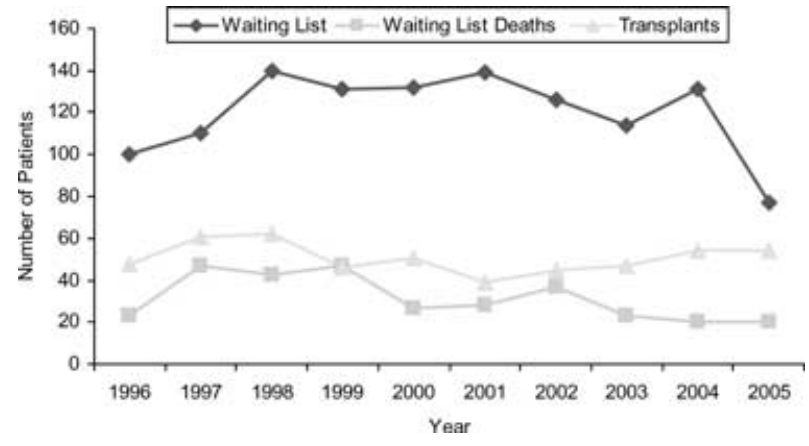

Source: 2006 OPTN/SRTR Annual Report, Tables 12.1a, 12.3$12.4 \mathrm{~b}$.

Figure 27: Pediatric patients listed for lung transplant, dying on the waiting list and transplanted, 1996-2005.

atric heart-lung transplantation seem to better reflect those seen in isolated pediatric lung transplantation than those in isolated heart transplantation. Since this procedure is performed at so few centers, it is also likely that trends in outcomes are related to the clinical practices and results at the small subgroup of centers that perform a high volume of cases (i.e. three or more cases per year).

\section{Waiting list}

Heart-lung transplantation remains a relatively rare procedure, with comparatively poor short- and long-term results. During the past 10 years, the number of new pediatric heart-lung registrants has decreased steadily, with only 13 new registrations in 2005 . This has resulted in a gradual decline in the number of patients on the waiting list. At the end of 2005, only eight children were active on the heartlung waiting list. The reasons are likely multifactorial and include use of bilateral lung transplantation as the procedure of choice for children with parenchymal lung disease and primary pulmonary hypertension. The relatively high death rate on the waiting list and poor long-term survival following transplantation may also discourage referral for consideration of transplantation.

\section{Transplantation and survival}

Between 1996 and 2004, 67 pediatric heart-lung transplants have been performed, with only 42 (63\%) having a functioning graft at the time of discharge and 35 (52\%) having a functioning graft 1 year following transplantation (SRTR analysis, May 2006). Only five such procedures were performed in 2005, three of which had a functioning graft at discharge.

\section{Lung Transplantation}

\section{Waiting list}

The numbers of pediatric lung candidates, deaths on the waiting list and transplants per year have been steady over the past decade, except for a large drop in the number of 


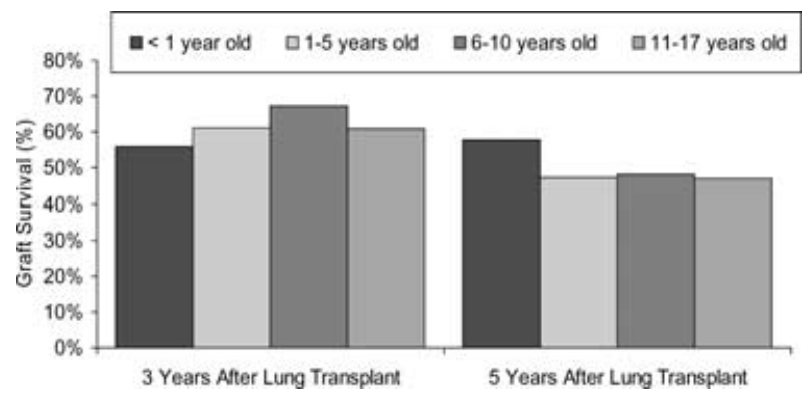

Source: 2006 OPTN/SRTR Annual Report, Table 12.10a.

Figure 28: Unadjusted graft survival at 3 and 5 years for deceased donor lung transplant recipients, by age.

pediatric candidates over the past year, from 131 in 2004 to 77 in 2005 (Figure 27). While the total number of deceased donor lung transplants has grown $78 \%$ over the decade, from 791 in 1996 to 1407 in 2005, the number of transplants in pediatric candidates showed no consistent trend. Consequently, the proportion of lung transplants to pediatric candidates has decreased: $4 \%$ of the recipients were children in 2005 compared with $5 \%$ in 1996. In 2005 , 53 pediatric candidates received lung transplants and 20 died waiting. The time until $25 \%$ of new registrants have received a lung transplant has varied widely among pediatric candidates over the past decade: between 17 and 156 days for registrants less than 1 year old, 39 and 279 days for those $1-5$ years, 54 and 883 days for children $6-10$ years and 110 and 783 days for adolescents aged $11-17$ years.

\section{Transplantation and survival}

At 3 years following transplantation, children aged 6-10 years had the best unadjusted graft survival among pediatric age groups at $73 \%$, followed by $1-5$-year-olds and infants less than 1 year at $61 \%$ and finally 11-17year-olds at $56 \%$ (Figure 28). Unadjusted graft survival at 5 years showed a similar pattern: $56 \%$ among infants less than 1 year, 68\% among 6-10-year-olds, 38\% among 1-5year-olds and $32 \%$ among 11-17-year-olds. At the end of 2004, 225 (4\%) of the 5139 people alive with functioning lung transplants had received their transplants as children (Figure 24).

\section{Immunosuppression}

Induction immunosuppression: The use of an induction regimen in pediatric lung transplant recipients and the types of agents used have changed over the last decade, with a general increase starting in 1999 (SRTR analysis, May 2006) (Figure 29). Center-specific practice patterns, recipient comorbidities, concerns for infectious complications (particularly CMV and fungal infection) and the risk of developing PTLD often have been cited as the primary influences on the use of specific agents. During the past decade, the percentage of pediatric lung recipients receiving an induction agent rose from 0\% in 1997 to a decade

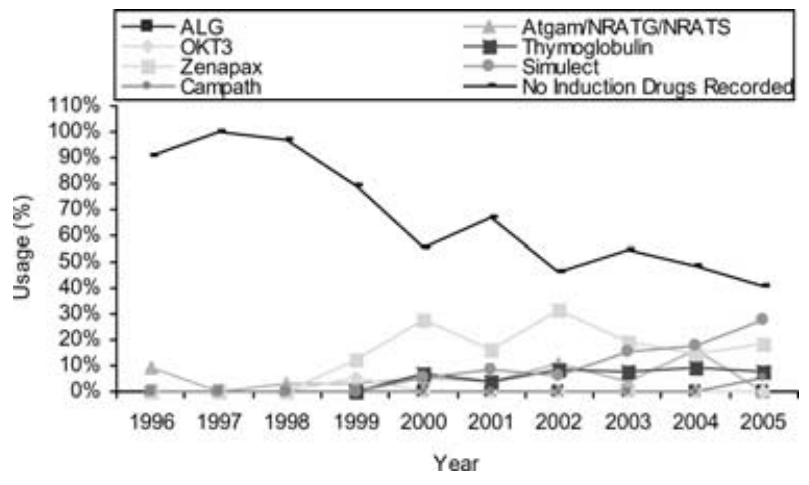

Source: SRTR Analysis, May 2006.

Figure 29: Immunosuppression use for induction for pediatric recipients with lung transplants, 1996-2005.

high of $59 \%$ in 2005 , in large part because of the availability of newer agents. Prior to 1999, anti-thymocyte globulin was the most commonly used induction agent. The use of rabbit anti-thymocyte globulin increased from $0 \%$ in 1999 to $17 \%$ in 2004 , declining to $12 \%$ of all patients receiving induction in 2005 . The most dramatic and steady increase is seen with the anti-IL-2R antibodies, daclizumab and basiliximab; use grew from $0 \%$ in 1998 to $78 \%$ of all pediatric patients receiving induction in 2005. Basiliximab is currently the single most commonly used induction agent in pediatric lung transplantation, accounting for $47 \%$ of all induction used. Use of alemtuzumab is first reported in 2005 , accounting for $9 \%$ (3 of 54) of all pediatric patients receiving induction.

Maintenance immunosuppression: Over the past 10 years, there has been a shift in the agents used for maintenance immunosuppressive therapy prior to discharge (SRTR analysis, May 2006) (Figure 30). Cyclosporine-based regimens have decreased, from $78 \%$ in 1996 to $49 \%$ in 2005. Conversely, use of tacrolimus-based regimens has

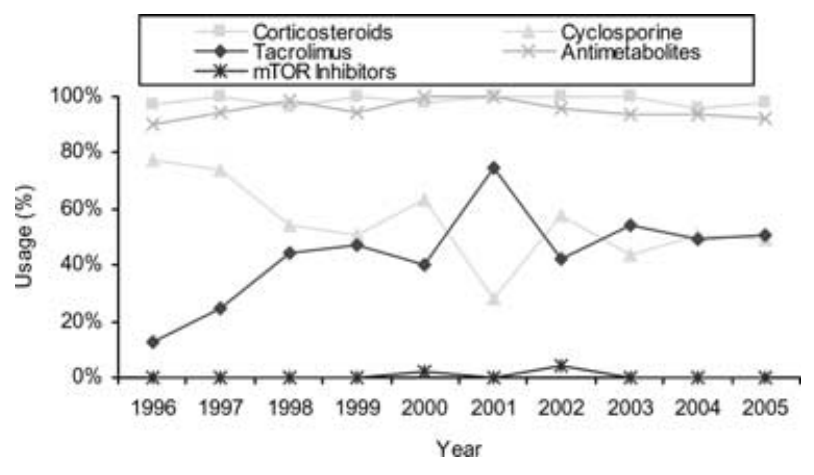

Source: SRTR Analysis, May 2006.

Figure 30: Immunosuppression use for maintenance of pediatric recipients with lung transplants prior to discharge, 19962005. 
increased over the same period, from $12 \%$ in 1996 to $51 \%$ in 2005. Azathioprine use has decreased even more, from $82 \%$ in 1996 to $31 \%$ in 2005, while MMF has increased from less than $8 \%$ in 1996 to $61 \%$ in 2005 . Sirolimus use before discharge was first reported in 2000 (1 patient of 52 ), peaked at $4 \%$ (2 of 45) in 2002, and then declined back to $0 \%$ from 2003 to the present, in response to safety issues associated with impairment of bronchial anastomotic healing. In contrast with recent trends among other solid organs, corticosteroids are still used for maintenance prior to discharge in virtually all patients (98\% in 2005), as they have been for the last 10 years.

At the time of discharge in 2005, the single most common regimen was the combination of tacrolimus, MMF and steroids in $33 \%$ of pediatric recipients, followed by cyclosporine, MMF and steroids in $28 \%$ of such patients (SRTR analysis, May 2006). Cyclosporine combined with azathioprine and steroids, by far the most common regimen through the 1980s and mid-1990s (65\% in 1996), is now used for maintenance prior to discharge in $20 \%$ of patients.

In the most recent cohort from 2004, by 1 year after transplantation use of tacrolimus-based regimens increased to $64 \%$ while use of cyclosporine-based regimens decreased to 36\% (SRTR analysis, May 2006). Of these tacrolimus-based regimens, tacrolimus/MMF remains the single most commonly used combination. Sirolimus as part of the regimen was used in $5 \%$ of patients at 1 year following transplantation in the 2004 cohort.

Anti-rejection treatment: The trend has been toward reduced use of all types of anti-rejection therapy (SRTR analysis, May 2006). In 2004, 17 patients of a cohort of 57 received anti-rejection therapy (30\%, down from a 10-year high of $58 \%$ in 2000) during the first year after transplant. As with heart transplantation, this trend may reflect a true decrease in acute rejection rates associated with more modern induction and maintenance regimens. Likewise, it is conceivable that more rejection episodes are being treated with only a change in the maintenance regimen; decreased rates of rejection may represent under-reporting of rejection episodes as measured by the use of anti-rejection therapy. Finally, use of fewer surveillance biopsies in many lung transplant programs may yield a lower detection rate and thus a falsely low reported rejection rate.

The great majority of patients (varying from $88 \%$ to $100 \%$ over the past 10 years) with reported anti-rejection therapy received corticosteroids as treatment for the episode (SRTR analysis, May 2006). In 2003, 23\% of patients treated for rejection received an anti-lymphocyte antibody preparation, the most common agent used being horse anti-thymocyte globulin. Of interest, 15\% (2 of 13) of the antibody-treated group received an anti-IL-2R antibody, despite little data supporting the use of this class of agents for the treatment of rejection.

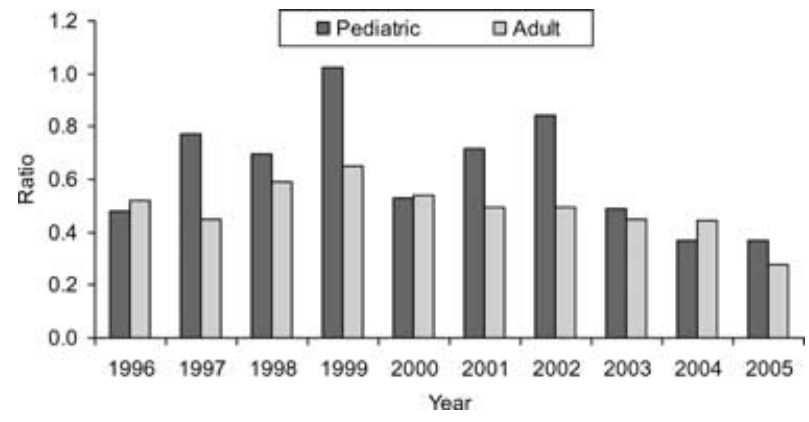

Source: 2006 OPTN/SRTR Annual Report, Tables 12.3-12.4b.

Figure 31: Ratio of lung waiting list deaths to transplants, pediatric and adult, 1996-2005.

\section{Recent changes in lung allocation}

Waiting list mortality in the pediatric lung population historically has been high, as have waiting list deaths compared with adults (Figure 31). A significant change in allocation policy for deceased donor lungs was implemented in the United States in May 2005. Formerly an allocation system based primarily on waiting time, the new system now uses the concepts of transplant benefit and medical urgency as the principal mechanisms by which to distribute lungs to transplant candidates aged 12 years and older (albeit with geography still having a major effect); allocation policy did not change for younger candidates.

The new allocation system is designed to maximize the 1-year survival benefit of lung transplantation by incorporating a prediction of the difference between measures of waiting list survival and post-transplant survival for each candidate. An additional goal is to minimize deaths on the waiting list by balancing the benefit calculation and the degree of medical urgency, as embodied in the waiting list survival measure. Four main categories of diagnosis were found to be strongly associated with waiting list and posttransplant mortality. These include Group A: obstructive lung diseases, typified by chronic obstructive pulmonary disease; Group B: pulmonary vascular diseases, principally primary pulmonary hypertension; Group C: cystic fibrosis and immunodeficiency disorders; and Group D: restrictive lung diseases, mainly idiopathic pulmonary fibrosis. About $20 \%$ of candidates and recipients have diagnoses other than the four mentioned, and these were assigned into one of the four groups using a combination of pathophysiologic similarity and comparable waiting list mortality risk. A number of other factors were significant in these mortality models, some of which varied substantially by diagnosis group, either in magnitude or degree of importance. In these cases, appropriate interaction terms were included to allow the impact of a risk factor to differ across diagnosis group.

Group E is reserved for patients under 12 years, irrespective of diagnosis. Analyses of pediatric candidates and re- 
cipients demonstrated that adolescent mortality risk was very similar to that for adults. In contrast, children under 12 years represent a heterogeneous group of diagnoses, often with unpredictable natural histories. Predictive factors for mortality in younger children-for which the numbers are much smaller, thus making modeling more difficulthave yet to be adequately determined. Therefore, allocation of donor lungs for these patients continues to be based on waiting time. For candidates 12 years and older, patients are ranked by an allocation score that is calculated as the difference between the transplant benefit measure (posttransplant survival measure minus the waiting list urgency measure) and the waiting list (urgency) measure. Mathematically, the value of this raw allocation score can range from -730 to +365 . To facilitate understanding, the raw allocation score is normalized to a scale from 0 to 100 and is referred to as the lung allocation score (LAS).

In an attempt to improve access to lungs for pediatric and adolescent recipients, a preferential allocation of pediatric and adolescent donor lungs to an age-matched recipient cohort has been incorporated as part of the new system. Lungs from donors aged less than 12 years are allocated to candidates aged less than 12 years first (based on candidate waiting time), then to candidates between 12 and 17 years (based on the LAS) and then last to candidates older than 18 years (again based on LAS). Lungs from donors aged 12-17 years are allocated to candidates between 12 and 17 years (based on LAS), then to candidates less than 12 years (based on waiting time) and then last to candidates older than 18 years (again based on LAS). Finally, lungs from donors older than 18 years are allocated to all candidates older than 12 years (based on LAS) before being offered to candidates less than 12 years (based on waiting time).

The new allocation system was implemented on May 4, 2005, and preliminary data have been recently analyzed (SRTR analysis, April 2006). In the initial 11 months, the number of active candidates older than 12 years with a nonzero score has decreased from 1269 to 874, a decline of over $30 \%$, while the number of candidates younger than 12 years has remained constant. This alone is noteworthy; prior to the implementation of the LAS, the size of the lung waiting list had increased every year since its inception. As of March 29, 2006, the median LAS was 33.6. There is substantial overlap between the distributions of the LAS (10th to 90th percentiles) across the four major diagnosis groups. The median LAS is slightly higher for Groups C and $D$ compared with Groups $A$ and $B$. The distribution of diagnosis groups among transplant recipients has shifted substantially, as has the position on the match run since the LAS was implemented. Prior to May 2005, almost half of the transplants were in group $A$ and a third in group D. In the 6 months following institution of the LAS, the distribution reversed, with now almost half in group $D$ and a third in group $A$. The distributions for groups $B$ and $C$ have not substantially changed in the first 6 months. Prior to LAS implementation, the transplant recipient was on average (median) 11th on the match run list for the organ with a 90 th percentile of 143 . Subsequent to implementation of the LAS, the median position on the list (for the first 9 months after implementation) is 5th with a 90th percentile of only 61 . This translates into a considerable time savings for an OPO in lung placement.

Of great importance to the pediatric lung transplant community is the change that has occurred in the recipient and donor ages. Although the total number of lung transplants involving pediatric donors aged 0-11 years is essentially unchanged during the two time periods (27 from May 4, 2004, to January 31, 2005, and 28 from May 4, 2005, to January 31,2006$)$, more of these young donor lungs are being directed toward adolescent recipients than under the old system (32\% vs. 7\%, respectively) instead of going to recipients older than 18 years. This is a goal of the LAS system, and the additional priority for pediatric organs going to pediatric recipients is clearly working. The allocation of adolescent donor organs (donors aged 12-17 years) has also shifted toward adolescent recipients and away from adults, although not to as great a degree as for the young pediatric donor organs.

With relatively small numbers and little time for follow-up, it is too early to make any definitive conclusions regarding the effect of the LAS on death rates on the waiting list or in the first year after transplantation. Preliminary favorable results for death rates per 100 patient-years on the waiting list for candidates 12 years and older show 14.1 deaths/100 patient-years (May 4, 2004, to December 31, 2004) versus 12.3 deaths/100 patient-years (May 4, 2005, to December $31,2005)$ with all diagnostic groups combined. Of particular interest to the pediatric lung transplant community is the change in Group C (consisting predominantly of cystic fibrosis patients) from 16.1 deaths/100 patient-years to 12.0 deaths/100 patient-years. There were no changes in the death rate seen for group $B$ (which includes the pulmonary hypertension cohort).

A high degree of caution needs to be exercised in making conclusions when comparing results between eras or diagnosis groups, given possible differences in patient populations. The profile of patients added to the waiting list after the LAS was implemented appears to differ from that added in the immediate prior year. For candidates younger than 12 years, an even higher degree of caution needs to be exercised in making any conclusions. In addition to changing patient profiles, there are very small numbers of patients and deaths. With that taken into account, preliminary results show a drop from 10.6 to 6.9 deaths/100 patient-years for all diagnostic groups combined. While the individual risk of death on the waiting list is expected to decrease dramatically under the LAS system, we may in fact observe a higher death rate among listed patients. This is possible because the active waiting list population 
has shifted from less urgent patients interested in accruing waiting time to patients in immediate need of an organ. Also, urgent patients who, under the LAS system, might survive long enough to be offered an organ are joining the waiting list under the new system when they would not likely have done so with a system based on waiting time. Posttransplant outcomes also may reflect the more urgent patients with high benefit being selected for transplantation. Evaluating the impact of the LAS on mortality will require careful thought.

The LAS system is a work in progress. Central to the new algorithm is a plan to regularly review the predictive models for waiting list and posttransplant mortality and to update them as needed. It is anticipated that serial clinical data will be useful in identifying new factors that should be incorporated into the distribution algorithm and that serially collected patient data may affect the importance of factors identified as significant in the analyses. At least every 6 months, analyses will be performed to identify factors and to possibly modify their hazard ratios in the algorithm. Thus, as patients are transplanted and removed from the list and new patients are added, risk is assessed using the most recent cohort of patients. Equally important is a provision for updating candidate data while on the waiting list. The current LAS system is based on data obtained at the time of waiting list placement and at transplant, as serial data were not available during the LAS development phase. It is anticipated that incorporating more current information into the algorithm will be a major enhancement. The important predictors of mortality are likely to change over time in concert with progression of the patient's underlying pulmonary disorder. The current LAS system does not address the issues of geographic disparities in access to lungs, as there is still a strong effect of prioritizing lungs locally rather than via a zonal or regional sharing mechanism/algorithm. It is hoped that this will be incorporated with further modifications to the lung allocation system if 'local first' is eliminated, similar to the newly approved heart allocation zonal sharing proposal.

\section{Summary}

In distinction from the steady increase in pediatric candidates each year through the 1990s, the trend since 2001 has been for relative stability in the number of pediatric waiting list registrants and transplant recipients across the organ groups, with the exception of patients needing intestine transplantation. Improving outcomes in terms of graft and patient survival continue to be seen, in most cases exceeding that seen in adult transplant recipients. With improved survival, focus has increasingly shifted to longterm outcomes other than survival alone, such as growth, development and renal function in nonrenal transplant recipients. Trends in immunosuppressive therapy across all organs show a move toward increased use of induction agents, especially rabbit anti-thymocyte globulin and IL-2R antagonists. Tacrolimus, with or without MMF, is most frequently employed for maintenance immunotherapy. Also, a gradual advance of steroid-free protocols is evident for all organs, except lung.

Allocation systems continue to undergo modification, often radically as in the case of the LAS. While allocation of organs to children is often secondary to the vastly larger adult waiting lists, all concerned with pediatric organ transplant patients, not least the OPTN Pediatric Committee, endeavor to maintain and improve access of children with end-stage organ failure to lifesaving transplantation. Despite improvements, waiting list and postoperative mortality rates are still far from negligible. Further progress is needed and expected.

\section{Acknowledgment}

The Scientific Registry of Transplant Recipients is funded by contract number 234-2005-37009C from the Health Resources and Services Administration (HRSA), U.S. Department of Health and Human Services. The views expressed herein are those of the authors and not necessarily those of the U.S. Government. This is a U.S. Government-sponsored work. There are no restrictions on its use.

This study was approved by HRSA's SRTR project officer. HRSA has determined that this study satisfies the criteria for the IRB exemption described in the 'Public Benefit and Service Program' provisions of 45 CFR 46.101 (b)(5) and HRSA Circular 03.

Note on sources: The articles in this report are based on the reference tables in the 2006 OPTN/SRTR Annual Report, which are not included in this publication. Many relevant data appear in the figures included here; other tables from the Annual Report that serve as the basis for this article include the following: Tables 1.4, 1.10, 2.1-2.7, 5.1a, 5.2, 5.4a, 5.4c, 5.10a, 5.10c, 9.1a-b, 9.3, 9.4a-b, 9.5, 9.7a, 9.10a-b, 9.14a, 9.16, 10.1a, 10.2$10.5,10.10,10.14,10.16,11.1 a, 11.3,11.4,11.14,11.16,12.1 a, 12.2,12.3$ 12.4a-b, 12.10a, 12.16, 13.1b, 13.2-13.4, 13.15 and 15.3. All of these tables may be found online at: http://www.ustransplant.org

\section{References}

1. Colombani PM, Dunn SP, Harmon WE, Magee JC, McDiarmid SV, Spray TL. SRTR report on the state of transplantation: Pediatric transplantation. Am J Transplant 2003; 3(Suppl. 4): 53-63.

2. Magee JC, Bucuvalas JC, Farmer DG, Harmon WE, HulbertShearon TE, Mendeloff EN. SRTR report on the state of transplantation: Pediatric transplantation. Am J Transplant 2004; 4(Suppl. 9): 54-71.

3. Harmon WE, MacDonald RA, Reyes JD et al. SRTR report on the state of transplantation: Pediatric transplantation, 1994-2003. Am J Transplant 2005; 5(4 Pt 2): 887-903.

4. Sweet SC, Wong H-H, Webber SA et al. The 2005 SRTR report on the state of transplantation: pediatric transplantation in the United States, 1995-2004. Am J Transplant 2006; 6: 1132-1152.

5. Martin SR, Atkison P, Anand R, Lindblad AS, SPLIT Research Group. Studies of pediatric liver transplantation 2002: Patient and graft survival and rejection in pediatric recipients of a first liver transplant in the United States and Canada. Pediatr Transplant 2004; 8: 273-283. 
6. Seikaly M, Ho PL, Emmett L, Tejani A. The 12th annual report of the North American Pediatric Renal Transplant Cooperative Study: Renal transplantation from 1987 through 1998. Pediatr Transplant 2001; 5: 215-231.

7. Herrington CS, Tsirka AE. Pediatric cardiac transplantation. Semin Thorac Cardiovasc Surg 2004; 16: 404-409.

8. Sweet SC. Pediatric lung transplantation: Update 2003. Pediatr Clin North Am 2003; 50: 1393-1417.

9. Grant D, Abu-Elmagd K, Reyes J et al., on behalf of the Intestine Transplant Registry. 2003 Report of the intestine transplant registry: A new era has dawned. Ann Surg 2005; 241: 607-613.

10. Rianthavorn P, Al-Akash SI, Ettinger RB. Kidney Transplantation in Children, Weir MR, ed. Philadelphia: Lippincott, William and Wilkins, 2005, Chapter 14: 198-230.

11. NAPRTCS: North American Pediatric Renal Transplant Cooperative Study (NAPRTCS) 2006 Annual Report. Available online at www.naprtcs.org. (Accessed on September 14, 2006).

12. U.S. Renal Data System, USRDS 2005 Annual Data Report: Atlas of End-Stage Renal Disease in the United States. National Institutes of Health, National Institute of Diabetes and Digestive and Kidney Diseases, Bethesda, MD, 2005.

13. McDonald RA, Guidinger MK, McCullough KP et al. Simulation modeling of preferential allocation of kidneys from donors less than age 35 years to pediatric candidates (abstract). Am J Transplant 2005; 5(Suppl. 11): 281

14. Organ Procurement and Transplantation Network: Policies. http://www.optn.org/policiesAndBylaws/policies.asp (Accessed on September 12, 2006).

15. Gritsch HA, McDonald R, Leichtman A et al. Should pediatric patients wait for an HLA-DR matched kidney? (abstract) Am J Transplant 2005; 5(Suppl. 11): 401

16. Dobbels F, Van Damme-Lombaert R, Vanhaecke J, De Geest S. Growing pains: Non-adherence with the immunosuppressive regimen in adolescent transplant recipients. Pediatr Transplant 2005; 9: 381-390.

17. Rianthavorn $P$, Ettenger RB, Malekzadeh M, Marik J, Struber M. Noncompliance with immunosuppressive medications in pediatric and adolescent patients receiving solid-organ transplants. Transplantation 2004; 77: 778-782.

18. Watson AR. Non-compliance and transfer from paediatric to adult transplant unit. Pediatric Nephrol 2000; 14: 469-472.

19. Magee JC, Thomas SE, Fredericks EM et al. Effect of recipient age and 'transition' on graft loss in pediatric transplant recipients (abstract). Am J Transplant (joint supplement for WTC, July 2006): 214.
20. Shneider BL, Neimark E, Frankenberg T, Arnott L, Suchy FJ, Emre $\mathrm{S}$. Critical analysis of the pediatric end-stage liver disease scoring system: A single center experience. Liver Transpl 2005; 11: 788795.

21. Shneider BL, Suchy FJ, Emre S. National and regional analysis of exceptions to the pediatric end-stage liver disease scoring system (2003-2004). Liver Transpl 2006; 12: 40-45.

22. Rodriguez-Luna $H$, Vargas HE, Moss A, Reddy KS, Freeman RB, Mulligan D. Regional variations in peer reviewed liver allocation under the MELD system. Am J Transplant 2005; 5: 22442247.

23. Salvalaggio PR, Neighbors $K$, Kelly $S$ et al. Regional variation and use of exception letters for cadaveric liver allocation in children with chronic liver disease. Am J Transplant 2005; 5: 18681874.

24. Gish RG, ed. Message meeting: MELD Exception Study Group. Liver Transpl 2006; 12(Suppl. 3): S85-S136.

25. Magee JC, Feng S. PELD: Working well, but only half of the time? Am J Transplant 2005; 5: 1785-1786.

26. Merion RM, Schaubel DE, Dykstra DM, Freeman RB, Port FK, Wolfe RA. The survival benefit of liver transplantation. Am J Transplant 2005; 5: 307-313.

27. Merion RM, Rush SH, Dykstra DM, Goodrich N, Freeman RB Jr, Wolfe RA. Predicted lifetimes for adult and pediatric split liver versus adult whole liver transplant recipients. Am J Transplant 2004; 4: 1792-1797.

28. Roberts JP, Hulbert-Shearon TE, Merion RM, Wolfe RA, Port FK. Influence of graft type on outcomes after pediatric liver transplantation. Am J Transplant 2004; 4: 373-377.

29. 2003 Annual Report of the U.S. Organ Procurement and Transplantation Network and the Scientific Registry of Transplant Recipients: Transplant Data 1993-2002. Department of Health and Human Services, Health Resources and Services Administration, Office of Special Programs, Division of Transplantation, Rockville, MD; United Network for Organ Sharing, Richmond, VA; University Renal Research and Education Association, Ann Arbor, MI.

30. Horslen SP. Optimal management of the post-intestinal transplant patient. Gastroenterology 2006; 130(2 Suppl. 1): S163S169.

31. Lee CK, Christensen LL, Magee JC, Ojo AO, Harmon WE, Bridges ND. Chronic renal dysfunction after pediatric heart transplantation: A 10-year national cohort study. J Heart Lung Transplant (in press).

32. West LJ, Pollock-Barziv SM, Dipchand Al et al. ABO-incompatible heart transplantation in infants. N Engl J Med 2001; 344: 793800. 